\title{
MEHLER SEMIGROUPS, ORNSTEIN-UHLENBECK PROCESSES AND BACKGROUND DRIVING LÉVY PROCESSES ON LOCALLY COMPACT GROUPS AND ON HYPERGROUPS
}

\author{
Wilfried Hazod
}





\title{
MEHLER SEMIGROUPS, ORNSTEIN-UHLENBECK PROCESSES AND BACKGROUND DRIVING LÉVY PROCESSES ON LOCALLY COMPACT GROUPS AND ON HYPERGROUPS
}

\author{
WILFRIED HAZOD
}

\begin{abstract}
For finite dimensional vector spaces it is well-known that there exists a 1-1-correspondence between distributions of Ornstein-Uhlenbeck type processes (w.r.t. a fixed group of automorphisms) and (background driving) Lévy processes. An analogous result could be proved for simply connected nilpotent Lie groups. Here we extend this correspondence to a class of commutative hypergroups.
\end{abstract}

\section{INTRODUCTION}

Let $\mathbb{V}$ be a $d$-dimensional real vector space and let $\left(T_{t}\right)_{t \in \mathbb{R}}$ be a continuous one-parameter group of automorphisms. M-semigroups (or skew semigroups) are continuous one-parameter families of probabilities $(\mu(t))_{t \geq 0}$ on $\mathbb{V}$ satisfying $\mu(t+s)=\mu(t) \star T_{t}(\mu(s)), \forall s, t \geq 0$. These skew or M-semigroups are distributions of (generalized) Ornstein-Uhlenbeckprocesses (resp. Mehler semigroups of transition kernels) and correspond in a 1-1-manner to continuous convolution semigroups, the distributions of Lévy processes (called background driving Lévy processes). The correspondence is expressed by path-wise random integral representations of the involved processes. See [25] for $d=1$, [2] or [32] and the literature mentioned there. More generally, for random integrals of additive processes see [37]. It should be mentioned that limits of M-semigroups are self-decomposable laws and vice versa. For the background of self-decomposability and random integral representations on vector spaces see e.g. the monograph [26], or [39, 28, 27], furthermore, $[1,38,37]$, and the literature mentioned there. For some applications of self-decomposability see e.g., [4, 29] and the references there.

For locally compact groups $\mathbb{G}$ admitting a continuous one-parameter group $\left(T_{t}\right)_{t \in \mathbb{R}} \subseteq \operatorname{Aut}(\mathbb{G})$, Ornstein-Uhlenbeck processes (or Mehler semigroups of transition kernels) resp. M-semigroups on the one side and Lévy processes resp. continuous convolution semigroups on the other, are defined verbatim as in the vector space case. In the group case - as random integral representations are in general not available - at least for contractible simply connected nilpotent Lie groups a 11-correspondence between M-semigroups and continuous convolution semigroups is established via Lie-Trotter product formulas

$$
(L T 1) \quad \mu(t)=\lim _{n \rightarrow \infty} \underset{k=0}{\star n-1} \underset{\frac{k t}{n}}{\star}\left(\mu_{t / n}\right) \quad(L T 2) \quad \mu_{t}=\lim _{n \rightarrow \infty} \mu(t / n)^{n}
$$

which may be understood as weak versions of random integral representations. See e.g., [14], §2.14, [16], Theorem C, [15]. (For a processapproach under some technical conditions see e.g., [30].)

Date: Version 14.12.08. 
The proof relies (i) on the construction of (space-time-) Lévy processes resp. continuous convolution semigroups on the space-time building $\Gamma:=\mathbb{G} \rtimes \mathbb{R}$, (ii) on the existence of common cores for generators of continuous convolution semigroups and (iii) on Lie-Trotter formulas for addition of generators of $C_{0}$-contraction semigroups. The second property, the existence of common cores, proved independently and nearly simultaneously by J. Faraut, K. Harzallah, F. Hirsch, J.P. Roth, $[12,11,21,22,23,24,35]$, is crucial. See also [13, 8, 9, 19]. (In fact, for our purpose a slight generalization of this result is needed, see Theorem 1.9 b), c) below.)

As a corollary it follows that the Bruhat test functions $\mathcal{D}(\mathbb{G})$ and for direct and semidirect extensions $\Gamma=\mathbb{G} \rtimes \mathbb{R}$ - that the subspaces $\mathcal{D}(G) \otimes \mathcal{D}(\mathbb{R}) \subseteq \mathcal{D}(\Gamma)$ are common cores for generators of continuous convolution semigroups on $\mathbb{G}$ and $\Gamma$ respectively. A key result which enables e.g. to verify $(L T 1)$ and $(L T 2)$. (Recall that for Lie groups $\mathcal{D}(\mathbb{G})$ is just $C_{c}^{\infty}(\mathbb{G})$.)

Recently M. Rösler [36] and M. Voit [40] investigated hypergroup structures on the cone of non-negative definite $d \times d$-matrices with a group like behaviour. In particular, the structure of the automorphism group is well-known, a homomorphic image of $\mathrm{GL}\left(\mathbb{R}^{d}\right)$. In fact, for $a \in \mathrm{GL}\left(\mathbb{R}^{d}\right)$ there corresponds an automorphism $\mathcal{K} \ni \kappa \mapsto T_{a}(\kappa):=$ $\left(a \kappa^{2} a^{*}\right)^{1 / 2} \in \mathcal{K}$. In [17] some probabilistic aspects of these hypergroup structures were investigated, especially divisibility, (semi-)stability and also self-decomposability and M-semigroups. However, the problem of existence of background driving Lévy processes and the correspondence by Lie-Trotter formulas was not investigated there. This is the main target of the present investigations.

Note that a version of the above-mentioned theorem of F. Hirsch et al. for hypergroups is proved in the thesis S. Menges [33], 5.26. There also the existence of a common core for convolution semigroups on commutative hypergroups is established ([33], 5.17, 5.22). However, for non-Abelian hypergroups there is no natural candidate for a common core as e.g., $\mathcal{D}(\mathbb{G})$ for general locally compact groups. To find such function spaces on semi-direct extensions and to show a core property which allows to prove the analogues of $(L T 1)$ and $(L T 2)$ is a crucial tool of this investigation.

In Section 1 we collect notations and basic facts for continuous convolution semigroups and invariant $C_{0}$-contraction semigroups, including a sketch of the afore mentioned Theorem of F. Hirsch et al. (in its slightly generalized form.) In Section 2 we apply these results to the case of locally compact groups (generalizing slightly the already published results for nilpotent Lie groups). Section 3 contains the main results: Theorem 3.1 and 3.2. The proof of the first is a consequence of the results collected in Section 2, whereas Section 4 is concerned with the proof of Theorem 3.2, the hypergroup case: For a class of hypergroups containing the afore mentioned hypergroups on matrix cones the existence of background driving Lévy processes and the correspondence via the Lie-Trotter formulas is established. The proof is quite technical and sometimes cumbersome, but I was unable to find a more elegant version. 


\section{Notations And BASic faCts}

Let $\mathbb{G}$ be a locally compact group or a hypergroup. (Or a locally compact semigroup with unit $e$ and with a nice behaviour at $\infty$ : for all compact $M, N \subseteq \mathbb{G}$ the set $\{z \in \mathbb{G}: \forall x \in N x z$ or $z x \in M\}$ is relatively compact.) According to the Riesz representation theorem measures $\mu \in \mathcal{M}^{b}(\mathbb{G})$ are identified with continuous linear functionals on $C_{0}(\mathbb{G})$, the dual pairing is denoted by $\int_{\mathbb{G}} f d \mu=\langle f, \mu\rangle$.

Measures are also identified with linear operators, the convolution operators acting e.g. on $C_{0}(\mathbb{G})$ from right resp. left:

$$
\begin{array}{ll}
R_{\mu}: & \left(R_{\mu} f\right)(x):=\int f d\left(\varepsilon_{x} \star \mu\right)=\left\langle f, \varepsilon_{x} \star \mu\right\rangle \\
L_{\mu}: & \left(L_{\mu} f\right)(x):=\int f d\left(\mu \star \varepsilon_{x}\right)=\left\langle f, \mu \star \varepsilon_{x}\right\rangle
\end{array}
$$

In particular, for $\mu=\varepsilon_{x_{0}}$ we use the abbreviations $R_{x_{0}}:=R_{\varepsilon_{x_{0}}}$ resp. $L_{x_{0}}:=L_{\varepsilon_{x_{0}}}$ for the right and left translations.

We collect some well-known properties of convolution operators which are tacitly used in the sequel. ( See e.g., [18, 13], and for hypergroups, [5].)

Proposition 1.1. a) $R_{\mu}$ and $L_{\mu}$ are linear operators acting on $C_{0}(\mathbb{G})$ with $\left\|R_{\mu}\right\|_{\infty}=\left\|L_{\mu}\right\|_{\infty}=\|\mu\|_{\infty}$

b) $R_{\mu} L_{\nu}=L_{\nu} R_{\mu} \quad$ for all $\mu, \nu \in \mathcal{M}^{b}(\mathbb{G})$

c) $R_{\mu \star \nu}=R_{\mu} R_{\nu}$ and $L_{\mu \star \nu}=L_{\nu} R_{\mu}$ for all $\mu, \nu \in \mathcal{M}^{b}(\mathbb{G})$

d) $\langle f, \mu \star \nu\rangle=\left\langle R_{\mu} f, \nu\right\rangle=\left\langle L_{\nu} f, \mu\right\rangle \quad \forall \mu, \nu \in \mathcal{M}^{b}(\mathbb{G}), f \in C_{0}(\mathbb{G})$

In particular, for $\nu=\varepsilon_{e}$ resp. $=\varepsilon_{x_{0}}$

d1) $\langle f, \mu\rangle=R_{\mu} f(e)=L_{\mu} f(e) \quad \forall \mu \in \mathcal{M}^{b}(\mathbb{G}), f \in C_{0}(\mathbb{G})$

d2) $f\left(x_{0}\right)=\left\langle f, \varepsilon_{x_{0}}\right\rangle=R_{x_{0}} f(e)=L_{x_{0}} f(e) \quad \forall f \in C_{0}(\mathbb{G})$

d3) $R_{\mu} f\left(x_{0}\right)=\left\langle f, \varepsilon_{x_{0}} \star \mu\right\rangle=\left\langle R_{\mu} f, \varepsilon_{x_{0}}\right\rangle=\left\langle L_{x_{0}} f, \mu\right\rangle=\left\langle L_{x_{0}} R_{\mu} f, \varepsilon_{e}\right\rangle$ $\forall \mu, \nu \in \mathcal{M}^{b}(\mathbb{G}), f \in C_{0}(\mathbb{G}), x_{0} \in \mathbb{G}$

d4) $L_{\mu} f\left(x_{0}\right)=\left\langle f, \mu \star \varepsilon_{x_{0}}\right\rangle=\left\langle L_{\mu} f, \varepsilon_{x_{0}}\right\rangle=\left\langle R_{x_{0}} f, \mu\right\rangle=\left\langle R_{x_{0}} L_{\mu} f, \varepsilon_{e}\right\rangle$ $\forall \mu, \nu \in \mathcal{M}^{b}(\mathbb{G}), f \in C_{0}(\mathbb{G}), x_{0} \in \mathbb{G}$

Proposition 1.2. Let $f \in C_{0}(\mathbb{G})$, and let $x_{0} \in \mathbb{G}$ such that $\left|f\left(x_{0}\right)\right|=$ $\|f\|_{\infty}$. Then $\|f\|_{\infty}=\left|R_{x_{0}} f(e)\right|=\left\|R_{x_{0}} f\right\|_{\infty}$

$\| R_{x_{0}}$ is a contraction (Proposition $1.1 \mathrm{a}$ ) ), hence $\left\|R_{x_{0}} f\right\|_{\infty} \leq\|f\|_{\infty}$. On the other hand, according to property d2) in Proposition 1.1, $\left|f\left(x_{0}\right)\right|$ $=\left|\left\langle R_{x_{0}} f, \varepsilon_{e}\right\rangle\right|$, whence $\left.\|f\|_{\infty}=\left|f\left(x_{0}\right)\right|=\left|R_{x_{0}} f(e)\right| \leq\left\|R_{x_{0}} f\right\|_{\infty} \quad\right]$

Let $T:=R_{\lambda}, \lambda \in \mathcal{M}^{b}(\mathbb{G}) . T$ is left invariant, i.e. $T L_{x}=L_{x} T \forall x \in \mathbb{G}$ (see Proposition 1.1) and $\langle f, \lambda\rangle=T f(e), T f(x)=\left\langle L_{x} f, \lambda\right\rangle$. This is a motivation to define

Definition 1.3. A subspace $\mathbb{D} \subseteq C_{0}(\mathbb{G})$ is called left invariant if $L_{x} \mathbb{D} \subseteq$ $\mathbb{D}, \forall x \in \mathbb{G}$, and a linear operator $U: \mathbb{D} \rightarrow C_{0}(\mathbb{G})$ is called left invariant if $\mathbb{D}$ is left invariant and $U L_{x}=L_{x} U \forall x \in \mathbb{G}$. Hence $U L_{\nu}=L_{\nu} U$ for all $\nu \in \mathcal{M}^{b}(\mathbb{G})$ with $L_{\nu}(\mathbb{D}) \subseteq \mathbb{D}$.

In this case, we define the linear functional $A: \mathbb{D} \rightarrow \mathbb{C}$ by $\langle f, A\rangle:=$ $T f(e)$, hence (according to 1.1. d2) ) $U f(x)=L_{x} U f(e)=U L_{x} f(e)=$ $\left\langle L_{x} f, A\right\rangle$. This motivates the notation $U=R_{A}$ (in analogy to Proposition 1.1. d3)). 
Definition 1.4. Let $U: \mathbb{D} \rightarrow C_{0}(\mathbb{G})$ be a linear operator acting on a subspace $\mathbb{D} \subseteq C_{0}(\mathbb{G})$. $U$ is called dissipative if for all $f \in \mathbb{D}$, for all $x_{0} \in \mathbb{G}$ such that $f\left(x_{0}\right)=\|f\|_{\infty}$ it follows $\Re\left(U f\left(x_{0}\right)\right) \leq 0$.

Proposition 1.5. a) Let $\left(T_{t}\right)_{t \geq 0}$ be a $C_{0}$-contraction semigroup on $C_{0}(\mathbb{G})$ with infinitesimal generator $\left(U:=\left.\frac{d^{+}}{d t}\right|_{t=0} T_{t}, D(U)\right)$. Then the domain $D(U)$ is dense and $U$ is closed and dissipative. Furthermore, $(I-U) D(U)=C_{0}(\mathbb{G})$.

b) Conversely, let $U$ be dissipative with dense domain $\mathbb{D}$. Then $(U, \mathbb{D})$ is closable, and the closure $(\bar{U}, \overline{\mathbb{D}})$ is closed and dissipative. Furthermore, $(I-U)(\mathbb{D})$ is dense in $(I-\bar{U})(\overline{\mathbb{D}})$.

c) If in addition, $(I-U)(\mathbb{D})$ is dense in $C_{0}(\mathbb{G})$ then $(\bar{U}, \overline{\mathbb{D}})$ is the generator of a (uniquely determined) $C_{0}$-contraction semigroup $\left(T_{t}\right)_{t \geq 0}$.

In the latter case, $\mathbb{D}$ is called 'core' for the generator of $\left(T_{t}\right)_{t \geq 0}$.

【 This characterization of generators of contraction semigroups as dissipative operators is known as Theorem of Lumer-Phillips ([31]). ]

As a consequence of the Riesz representation theorem we obtain

Proposition 1.6. A left invariant linear operator $T=R_{A}-A$ defined as above in 1.3 - defined on $\mathbb{D}:=C_{0}(\mathbb{G})$ is the convolution operator of a bounded measure $A=\lambda \in \mathcal{M}^{b}(\mathbb{G})$, and conversely.

In particular, a $C_{0}$-semigroup of invariant operators on $C_{0}(\mathbb{G})$ is representable as $\left(T_{t}=R_{\lambda_{t}}\right)_{t \geq 0}$ where $\left(\lambda_{t}\right)_{t \geq 0}$ is a continuous convolution semigroup in $\mathcal{M}^{b}(\mathbb{G})$ with $\lambda_{0}=\varepsilon_{e}$.

We adopt the following notations: $\mathcal{M}^{1}(\mathbb{G})$ denotes the set of probability measures and $\mathcal{M}^{(1)}(\mathbb{G}):=\left\{\lambda \in \mathcal{M}^{b}(\mathbb{G}):\|\lambda\| \leq 1\right\}$.

In the sequel we shall always tacitly assume for continuous convolution semigroups that $\lambda_{0}=\varepsilon_{e}$. Let $\left(\lambda_{t}\right)_{t \geq 0}, \lambda_{0}=\varepsilon_{e}$, be a continuous convolution semigroup in $\mathcal{M}^{b}(\mathbb{G})$ with corresponding $C_{0}$-operator semigroup $\left(T_{t}=R_{\lambda_{t}}\right)_{t \geq 0}$. Then the infinitesimal generator $(U, D(U))$ is a left invariant operator. If moreover, $\left(\lambda_{t}\right) \subseteq \mathcal{M}^{(1)}(\mathbb{G})$ then $(U, D(U))$ is (left invariant and) dissipative.

In view of Popositions 1.5 and 1.6 we have:

Proposition 1.7. Let $(U, D(U))$ be left invariant and dissipative and assume $(I-U) D(U)=C_{0}(\mathbb{G})$, hence $U$ is the generator of a $C_{0}-$ contraction semigroup $\left(T_{t}\right)_{t \geq 0}$. Then $T_{t}=R_{\lambda_{t}}$ for some continuous convolution semigroup $\left(\lambda_{t}\right)_{t \geq 0} \subseteq \mathcal{M}^{(1)}(\mathbb{G})$.

IF For $\alpha>0$ the resolvent $I_{\alpha}:=\left(U-\frac{1}{\alpha} I\right)^{-1}$ is bonded, obviously left invariant, hence a convolution operator of a bounded measure. Any $T_{t}$ is representable as limit of exponentials of resolvent operators, hence is itself left invariant.

Remark 1.8. Let $\mathbb{D}$ be a core for the generator of a semigroup of convolution operators $\left(R_{\lambda_{t}}\right)_{t \geq 0}$. Then, by a slight abuse of language, we call $\mathbb{D}$ a core for the continuous convolution semigroup $\left(\lambda_{t}\right)_{t \geq 0}$.

Now we are ready to formulate the announced result of J. Faraut, K. Harzallah, F. Hirsch and J.P. Roth ([12, 11, 21, 22, 23, 24, 35]). We 
restrict to the case of continuous convolution semigroups with trivial idempotents $\lambda_{0}=\varepsilon_{e}$. As mentioned in the above cited literature, the results generalize easily to continuous convolution semigroups with non-trivial idempotents $\lambda_{0}$. (If $\lambda_{t} \geq 0$ then $\lambda_{0}=\omega_{K}$, a Haar measure on some compact sub-(hyper)group $K$ ).

Theorem 1.9. Let $\mathbb{D}$ be a dense linear subspace of $C_{0}(\mathbb{G})$.
a) Assume
(i) $L_{x} \mathbb{D} \subseteq \mathbb{D} \forall x \in \mathbb{G}$ and
(ii) $R_{x} \mathbb{D} \subseteq \mathbb{D} \forall x \in \mathbb{G}$

Let $U: \mathbb{D} \rightarrow C_{0}(\mathbb{G})$ be a left invariant and dissipative linear operator. Then the closure $(\bar{U}, \overline{\mathbb{D}})$ is the generator of a left invariant contraction semigroup $\left(T_{t}=R_{\lambda_{t}}\right)_{t \geq 0}$. I.e., $\mathbb{D}$ is a core for the continuous convolution semigroup $\left(\lambda_{t}\right) \subseteq \mathcal{M}^{(1)}(\mathbb{G})$.

b) More generally, (ii) may be replaced by (ii') $R_{x} \mathbb{D} \subseteq \overline{\mathbb{D}} \forall x \in \mathbb{G}$. c) Let $(U, D(U))$ be a dissipative, closed and left invariant operator. Assume $\mathbb{D} \subseteq D(U)$ to be left-invariant (i), and assume furthermore (ii") $R_{x} \mathbb{D} \subseteq D(U) \forall x \in \mathbb{G}$.

Then $(U, D(U))$ is the generator of a left invariant contraction semigroup $\left(R_{\lambda_{t}}\right)_{t>0}$ and $\widetilde{\mathbb{D}}:=\operatorname{span}\left\{R_{x} \mathbb{D}: x \in \mathbb{G}\right\}$ is a left- and right invariant core for $(U, D(U))$ (resp. for $\left.\left(\lambda_{t}\right)_{t \geq 0}\right)$.

The following sketch of a proof follows - with different notations - the lines of the proofs in [21, 22]. See also [13]. For hypergroups a proof (of a) ) is contained in the thesis [33], 5.26.

Condition (ii') is weaker than (ii), hence $b) \Rightarrow a$ ).

To prove b) we first note that

1. Condition (i) implies $L_{\nu} \mathbb{D} \subseteq \overline{\mathbb{D}} \forall \nu \in \mathcal{M}^{b}(\mathbb{G})$. In fact, approximating $\nu$ by measures $\nu_{n}$ with finite supports such that $L_{\nu_{n}} \rightarrow L_{\nu}$ in the strong operator topology and observing $L_{\nu_{n}} \mathbb{D} \subseteq \mathbb{D}$ for all $n$ yields $L_{\nu_{n}} f \rightarrow L_{\nu} f$ for $f \in \mathbb{D}$, and furthermore, $U L_{\nu_{n}} f=L_{\nu_{n}} U f \rightarrow L_{\nu} U f$. Hence $L_{\nu} f \in \overline{\mathbb{D}}$ and $\bar{U} L_{\nu} f=L_{\nu} U f$.

Analogously, $\forall g \in \overline{\mathbb{D}}$ we obtain $L_{\nu} g \in \overline{\mathbb{D}}$ and $\bar{U} L_{\nu} g=L_{\nu} \bar{U} g$. (This applies in particular for $f \in \mathbb{D}, g:=R_{x_{0}} f$.)

2. Let $\nu \in((I-U) \mathbb{D})^{\perp}$. Since $(I-U) \mathbb{D}$ is dense in $(I-\bar{U}) \overline{\mathbb{D}}$, we have $\nu \perp(I-\bar{U}) \overline{\mathbb{D}}$.

Let $f \in \mathbb{D}$, let $x_{0} \in \mathbb{G}$ such that $\left\|L_{\nu} f\right\|_{\infty}=\left|L_{\nu} f\left(x_{0}\right)\right|=\left|R_{x_{0}} L_{\nu} f(e)\right|$, i.e., for some $c$ with $|c|=1$ we have $\left\|L_{\nu} f\right\|_{\infty}=c \cdot L_{\nu} f\left(x_{0}\right)$. W.l.o.g. we may assume $c=1$, else replace $f$ by $c \cdot f$.

As $g:=R_{x_{0}} f \in \overline{\mathbb{D}}$ by assumption (ii') we have $0=\left\langle(I-\bar{U}) R_{x_{0}} f, \nu\right\rangle=\left\langle L_{\nu}(I-\bar{U}) g, \varepsilon_{e}\right\rangle=L_{\nu} g(e)-\bar{U} L_{\nu} g(e)=$ $R_{x_{0}} L_{\nu} f(e)-\bar{U} R_{x_{0}} L_{\nu} f(e)=\left\|R_{x_{0}} L_{\nu} f\right\|_{\infty}-\bar{U} R_{x_{0}} L_{\nu} f(e)$. Since $\left\|L_{\nu} f\right\|_{\infty}=$ $\left(R_{x_{0}} L_{\nu} f\right)(e)=\left\|R_{x_{0}} L_{\nu} f\right\|_{\infty}$ (cf. Proposition 1.2) and $\bar{U}$ is dissipative, we have $\Re \bar{U} R_{x_{0}} L_{\nu} f(e) \leq 0$. Therefore, $\left\|R_{x_{0}} L_{\nu} f\right\|_{\infty}=0$. According to property a) in 1.1, $\left\|L_{\nu} f\right\|_{\infty}=0$ follows. Since $\mathbb{D}$ is dense in $C_{0}(\mathbb{G})$ we have proved $\nu=0$.

3. Therefore, $(I-U) \mathbb{D}$ is dense in $C_{0}(\mathbb{G})$.

Assertion b) (and hence a) ) follows by Proposition 1.7 .

To prove c), put $\widetilde{\mathbb{D}}:=\operatorname{span}\left\{R_{x} \mathbb{D}: x \in \mathbb{G}\right\}$.

Claim: $\widetilde{\mathbb{D}}$ is a core for $(U, D(U))$. Hence $(U, D(U))$ is maximal dissipative and therefore a generator. 
$\llbracket$ Obviously, $\mathbb{D} \subseteq \widetilde{\mathbb{D}} \subseteq D(U)$. Hence $\widetilde{\mathbb{D}}$ is dense, by construction left and right invariant and therefore according to a), $\widetilde{\mathbb{D}}$ is a core for the closure of the restriction $(U, \widetilde{\mathbb{D}})$. Since $(U, D(U))$ is closed, we observe $\overline{\widetilde{\mathbb{D}}} \subseteq D(U)$, hence $(\bar{U}, \overline{\widetilde{\mathbb{D}}})=(U, D(U))$ and $(I-U) D(U)=C_{0}(\mathbb{G})$ since $(I-U) \widetilde{\mathbb{D}}$ is dense in $\left.C_{0}(\mathbb{G})\right]$

We obtain immediately the well known result:

Corollary 1.10. Let $\mathbb{G}$ be a locally compact group. Then the Bruhat test function space $\mathcal{D}(\mathbb{G})$ is a common core for all continuous convolution semigroups $\left(\lambda_{t}\right)_{t>0}$ in $\mathcal{M}^{(1)}(\mathbb{G})$, in particular, for continuous convolution semigroups of probabilities.

$\llbracket \mathcal{D}(\mathbb{G})$ is dense, left- and right-invariant and - according to the LévyKhinchin-Hunt representation $-\mathcal{D}(\mathbb{G})$ is contained in the domain of the generator of any continuous convolution semigroup. Cf. e.g., [18], 4.4.18, 4.5.8 for continuous convolution semigroups of probabilities, see e.g. $[8,9,12,10,11,13,42,43]$ for the more general case $\mathcal{M}^{(1)}(\mathbb{G})$. $]$

Corollary 1.11. Let $\mathbb{G}$ be an Abelian locally compact group or an Abelian hypergroup. Then the space of 'analytic vectors' $\mathcal{A}:=\left(L_{c}^{1}(\widehat{\mathbb{G}})\right)^{\vee}$ is a common core for all continuous convolution semigroups $\left(\lambda_{t}\right)_{t \geq 0}$ in $\mathcal{M}^{(1)}(\mathbb{G})$. (Here $L_{c}^{1}$ denotes the space of functions with compact support which are integrable on the dual $\widehat{\mathbb{G}}$ w.r.t. the Haar resp. Plancherel measure, and $\vee$ denotes the inverse Fourier transform.) Analogously, $C_{c}(\widehat{\mathbb{G}})^{\vee}$ and $L_{c}^{2}(\widehat{\mathbb{G}})^{\vee}$ share this property.

$\llbracket \mathcal{A}$ is dense and left- and right-invariant. Furthermore, for any $f \in \mathcal{A}$ and any continuous convolution semigroup $t \mapsto R_{\lambda_{t}} f=\left(\widehat{\lambda}_{t} \cdot \widehat{f}\right)^{\vee}=$ $\left(e^{t \cdot \psi} \cdot \widehat{f}\right)^{\vee}$ (with $\psi:=\log \widehat{\lambda_{1}}$ ) is analytic. Therefore in particular, $f$ is contained in the domain of the generator. For groups a proof is found in e.g. [7], for hypergroups see [33], 5.17, 5.22.

Remark 1.12. For later use we note that the cores $\mathcal{D}(\mathbb{G})$ and $\mathcal{A}$ constructed above in Corollary 1.10 resp. 1.11 are invariant under automorphisms of $\mathbb{G}$.

\section{Semidirect products $\Gamma=\mathbb{G} \rtimes \mathbb{R}$ : The Case of Locally COMPACT GROUPS}

Throughout in this Section $\mathbb{G}, \mathbb{G}_{i}$ denote locally compact topological groups.

First we note a further corollary to Theorem 1.9:

Corollary 2.1. Let $\mathbb{G}_{i}, i=1,2$ be locally compact groups with test function spaces $\mathcal{D}\left(\mathbb{G}_{1}\right), \mathcal{D}\left(\mathbb{G}_{2}\right)$ respectively. Then the subspace $\mathbb{D}:=$ $\mathcal{D}\left(\mathbb{G}_{1}\right) \otimes \mathcal{D}\left(\mathbb{G}_{1}\right) \subseteq \mathcal{D}\left(\mathbb{G}_{1} \otimes \mathbb{G}_{2}\right)$ is a common core for continuous convolution semigroups in $\mathcal{M}^{(1)}\left(\mathbb{G}_{1} \otimes \mathbb{G}_{2}\right)$. 
『 On the one hand, $\mathbb{D} \subseteq \mathcal{D}\left(\mathbb{G}_{1} \otimes \mathbb{G}_{2}\right) \subseteq D(U)$ for any generator $(U, D(U))$ of a continuous convolution semigroup as mentioned in Corollary 1.10. On the other hand, $\mathbb{D}$ satisfies the conditions (i) and (ii) of Theorem 1.9 a).

Now let $\mathbb{G}$ denote a locally compact group and let $\left(T_{t}\right)_{t \in \mathbb{R}} \subseteq \operatorname{Aut}(\mathbb{G})$ be a continuous one parameter group. The semidirect product $\Gamma=$ $\mathbb{G} \rtimes \mathbb{R}$ is the Cartesian product $\mathbb{G} \otimes \mathbb{R}$ equipped with the group operation $(x, s)(y, t):=\left(x T_{s}(y), s+t\right) . \Gamma$ is a locally compact group and hence $\mathcal{D}(\Gamma)$ is a common core for continuous convolution semigroups in $\mathcal{M}^{(1)}(\Gamma)$. First we have

Proposition 2.2. Let $\mathbb{G}$ be a Lie group. Then $\mathbb{D}:=\mathcal{D}(\mathbb{G}) \otimes \mathcal{D}(\mathbb{R}) \subseteq$ $\mathcal{D}(\Gamma)$ is a common core for continuous convolution semigroups in $\mathcal{M}^{1}(\Gamma)$

Proof: In contrast to the above mentioned Corollary 2.1 now the proof relies on the weaker assumption (ii') in Theorem $1.9 \mathrm{~b}$ ).

1. Left invariance (i) is obvious: For $\varphi \otimes \psi \in \mathcal{D}(\mathbb{G}) \otimes \mathcal{D}(\mathbb{R})$ we have

$$
L_{(y, t)}(\varphi \otimes \psi)(x, s)=\varphi\left(y T_{t}(x)\right) \cdot \psi(s+t)=: \varphi_{1}(x) \cdot \psi_{1}(s)
$$

Hence $L_{(y, t)}(\varphi \otimes \psi) \in \mathbb{D} \forall(y, t) \in \Gamma$

2. Condition (ii') is fulfilled:

Let $\left(U=R_{A}, D(U)\right)$ be the generator of $\left(R_{\lambda_{t}}\right)_{t \geq 0}$, with a continuous convolution semigroup $\left(\lambda_{t}\right)_{t \geq 0} \subseteq \mathcal{M}^{(1)}(\Gamma)$. According to Corollary 1.10 $\mathbb{D} \subseteq \mathcal{D}(\Gamma) \subseteq D(U)$. Let $(\bar{U}, \overline{\mathbb{D}})$ denote the closure of the restriction $(U, \mathbb{D})$.

We have to show for all $(y, t) \in \Gamma$ that $R_{(y, t)} \mathbb{D} \subseteq \overline{\mathbb{D}}$. In fact,

$$
R_{(y, t)}(\varphi \otimes \psi)(x, s)=\varphi\left(x T_{s}(y)\right) \cdot \psi(s+t)
$$

We fix $\varepsilon_{n}>0, \delta_{n}>0, s_{i}^{(n)} \in \mathbb{R}, i \leq i \leq N_{n}$. Let $\operatorname{supp} \psi \subseteq[a, b] \subseteq$ $\bigcup_{i=1}^{N_{n}}\left[s_{i}^{(n)}-\delta_{n}, s_{i}^{(n)}+\delta_{n}\right]$. Choose furthermore $\gamma_{i}^{(n)} \in \mathcal{D}(\mathbb{R})$ such that $\operatorname{supp} \gamma_{i}^{(n)} \subseteq\left[s_{i}^{(n)}-\delta_{n}, s_{i}^{(n)}+\delta_{n}\right], 0 \leq \gamma_{i}^{(n)} \leq 1$ and $\sum_{1}^{N_{n}} \gamma_{i}^{(n)} \equiv 1$ on $[a, b]$. Put $\psi_{i}^{(n)}:=\gamma_{i}^{(n)} \cdot \psi$. Let $\varepsilon_{n} \rightarrow 0$ and choose $\gamma_{i}^{(n)}$ and $\delta_{n}$ such that

$$
\left\|(x, s) \mapsto \sum_{i=1}^{N_{n}}\left(\varphi\left(x T_{s}(y)\right)-\varphi\left(x T_{s_{i}^{(n)}}(y)\right)\right) \cdot \psi_{i}^{(n)}(s+t)\right\|_{C_{0}^{(2)}(\Gamma)}<\varepsilon_{n}
$$

We have

$$
\begin{aligned}
& H(x, s):=R_{(y, t)}(\varphi \otimes \psi)(x, s)= \\
& \sum_{i=1}^{N_{n}}\left(\varphi\left(x T_{s}(y)\right)-\varphi\left(x T_{s_{i}^{(n)}}(y)\right)\right) \cdot \psi_{i}^{(n)}(s+t)+\sum_{i=1}^{N_{n}} \varphi\left(x T_{s_{i}^{(n)}}(y)\right) \cdot \psi_{i}^{(n)}(s+t) \\
& =: G_{n}(x, s)+F_{n}(x, s)
\end{aligned}
$$

By construction, $\left\|G_{n}\right\|_{C_{0}^{(2)}(\Gamma)} \rightarrow 0$, furthermore, $F_{n} \in \mathbb{D}, H \in \mathcal{D}(\Gamma) \subseteq$ $D(U)$ and $F_{n} \rightarrow H$ in $C_{0}(\Gamma)$. The Lévy-Khinchin-Hunt representation (cf. e.g., [18], 4.4.18, 4.5.8, [19], resp. [8, 12, 10, 11, 13, 42, 43]) yields that the restriction of the generator $U=R_{A}: C_{0}^{(2)}(\Gamma) \rightarrow C_{0}(\Gamma)$ is continuous. Whence $\left\|U G_{n}\right\|_{\infty} \rightarrow 0$ and $U F_{n} \rightarrow U H$.

Therefore we have $H_{n} \rightarrow H$ and $U H_{n} \rightarrow U H$, whence $H \in \overline{\mathbb{D}}$, as asserted.

3. Now the proof follows by Theorem $1.9 \mathrm{~b}$ ). 
In all examples we have in mind, the underlying group is a (simply connected, nilpotent) Lie group. Nevertheless it is worth to point out that this result is true for general locally compact groups $\mathbb{G}$ which admit a continuous one-parameter group of automorphisms $\left(T_{t}\right)_{t \in \mathbb{R}} \subseteq \operatorname{Aut}(\mathbb{G})$ :

Theorem 2.3. Let $\mathbb{G}$ be a locally compact group with $\left(T_{t}\right)_{t \in \mathbb{R}} \subseteq \operatorname{Aut}(\mathbb{G})$. We define as above the semidirect extension $\Gamma=\mathbb{G} \rtimes \mathbb{R}$ and put again $\mathbb{D}:=\mathcal{D}(\mathbb{G}) \otimes \mathcal{D}(\mathbb{R})$.

Let $\left(\lambda_{t}\right)_{t \geq 0} \subseteq \mathcal{M}^{(1)}(\Gamma)$ be a continuous convolution semigroup with generating functional $A$ resp. infinitesimal generator $\left(U=R_{A}, D(U)\right)$. Then $\mathbb{D}$ is a core for $\left(\lambda_{t}\right)_{t \geq 0}$ resp. for $\left(U=R_{A}, D(U)\right)$.

We sketch a proof:

$\mathbb{D}$ is dense in $C_{0}(\Gamma)$ and $\mathbb{D} \subseteq \mathcal{D}(\Gamma) \subseteq D(U)$. As before, it follows immediately that $\mathbb{D}$ is left invariant.

Claim: $R_{(y, t)} \mathbb{D} \subseteq \overline{\mathbb{D}}$. (Again $(\bar{U}, \overline{\mathbb{D}})$ denotes the closure of the restriction $(U, \mathbb{D})$.)

As in Proposition 2.2, let $\delta_{n} \rightarrow 0$, let $\varphi \otimes \psi \in \mathcal{D}(\mathbb{G}) \otimes \mathcal{D}(\mathbb{R})$, define as in proposition $2.2, H:=R_{(y, t)} \varphi \otimes \psi, \psi=\sum \psi_{i}^{(n)}$ and decompose as before $H=F_{n}+G_{n}$.

$\left(T_{t}\right)$ is a continuous one-parameter group. The connected component $\mathbb{G}_{0}$ is characteristic and $\mathbb{G} / \mathbb{G}_{0}$ is totally disconnected. Therefore, the induced automorphisms $\bar{T}_{t}$ act trivially on $\mathbb{G} / \mathbb{G}_{0}$.

Choose an open subgroup $\mathbb{G}_{1} \subseteq \mathbb{G}$ such that $\mathbb{G}_{1} / \mathbb{G}_{0}$ is compact. Then, (e.g., according to [14], 3.1.22) we have $\mathbb{G}_{1}=\lim \mathbb{G}_{1} / K^{\alpha}$ with compact normal $T_{t^{-}}$invariant subgroups $K^{\alpha}$. Hence $\Gamma_{1}:=\mathbb{G}_{1} \rtimes \mathbb{R}$ is an open subgroup of $\Gamma$ and $\Gamma_{1}=\lim \Gamma_{1} / L^{\alpha}$ with $L^{\alpha}=K^{\alpha} \otimes\{0\}$.

The Lévy-Khinchin-Hunt representation for general locally compact groups (cf. e.g., [18, 19] resp. [8, 12, 10, 11, 13, 42, 43]) yields that $A=B+\eta$ where $\eta$ is a bounded measure (a Poisson generator), and $B$ is supported by $\Gamma_{1}$. We have $U=R_{A}=R_{B}+R_{\eta}$ and, as $\eta$ is bounded, $\left\|R_{\eta} G_{n}\right\|_{\infty} \rightarrow 0$, and $R_{\eta} F_{n} \rightarrow R_{\eta} H$.

Hence w.l.o.g. we may assume that $\Gamma=\Gamma_{1}$ is Lie projective.

Since $\varphi \in \mathcal{D}(\mathbb{G})$ is constant on $K^{\alpha}$-cosets for some $K^{\alpha}$ and all functions involved are hence left $K^{\alpha}$-invariant, we may assume w.l.o.g. that $\mathbb{G}=\mathbb{G}_{1} / K^{\alpha}$ resp. $\Gamma=\Gamma_{1} / L^{\alpha}$. Thus the proof is reduced to the case of Lie groups, which was proved in Proposition 2.2.

Lie-Trotter formulas. We recall Lie-Trotter product formulas for addition of generators of $C_{0}$ semigroups and its applications to continuous convolution semigroups. For the background see e.g., P.R. Chernoff [6], 1.1, and the literature mentioned there. For continuous convolution semigroups see e.g., [13].

Proposition 2.4. a) The sum $U+V$ of generators of $C_{0}-$ contraction semigroups $(U, D(U))$ and $(V, D(V))$ defines a dissipative operator on $D(U) \cap D(V)$. If $D(U) \cap D(V)$ is a core for $\overline{U+V}$ (hence for the generator of a contraction semigroup) then the involved semigroups are related by the Lie-Trotter formula:

$$
e^{t(U+V)}=\lim _{n \rightarrow \infty}\left(e^{(t / n) U} e^{(t / n) V}\right)^{n}
$$

(Convergence in the strong operator topology.) 
b) Applying this to continuous convolution semigroups (resp. to the corresponding convolution operators) we obtain:

Let $\left(\mu_{t}\right)_{t>0},\left(\nu_{t}\right)_{t \geq 0} \subseteq \mathcal{M}^{(1)}(\mathbb{G})$ be continuous convolution semigroups in on a locally compact group $\mathbb{G}$. Let $\mathbb{D}$ be a common core for all continuous convolution semigroups (e.g., $\mathbb{D}=\mathcal{D}(\mathbb{G})$ ). Then the sum of the generators is at least defined on $\mathbb{D}$ and its closure generates a continuous convolution semigroup $\left(\lambda_{t}\right)_{t \geq 0}$. Furthermore, the Lie-Trotter formula for continuous convolution semigroups holds true:

$$
(L T *) \quad \lambda_{t}=\lim _{n \rightarrow \infty}\left(\mu_{t / n} \star \nu_{t / n}\right)^{n}
$$

\section{The MAIN RESUlts}

In the following we consider a sub-semigroup of $\mathcal{M}^{1}(\Gamma)$, defined as $\mathcal{M}_{*}^{1}(\Gamma):=\left\{\mu \otimes \varepsilon_{t}: \mu \in \mathcal{M}^{1}(\mathbb{G}), t \in \mathbb{R}\right\}$. (Analogously $\mathcal{M}_{*}^{(1)}(\Gamma)$, $\mathcal{M}_{*,+}^{(1)}(\Gamma), \mathcal{M}_{*}^{b}(\Gamma)$ etc. are defined). Recall the definition of an Msemigroup in the Introduction: A continuous family $(\mu(t))_{t>0} \subseteq \mathcal{M}^{1}(\mathbb{G})$ is a M-semigroup iff

$$
\mu(s+t)=\mu(s) \star T_{s}(\mu(t)) \text { for all } s, t \geq 0 .
$$

Obviously, $(\mu(t))_{t \geq 0}$ is a M-semigroup in $\mathcal{M}^{1}(\mathbb{G})$ iff $\left(\lambda_{t}:=\mu(t) \otimes \varepsilon_{t}\right)_{t \geq 0}$ is a continuous convolution semigroup in $\mathcal{M}_{*}^{1}(\Gamma)$. Furthermore, as immediately verified, for $f \in \mathbb{D}:=\mathcal{D}(\mathbb{G}) \otimes \mathcal{D}(\mathbb{R})$ the generator $U$ of $\left(R_{\lambda_{t}}\right)$ splits as $U f=(W+P) f$ (resp. $\left.W f=(U-P) f\right)$, with $W f=\left.\frac{d^{+}}{d t}\right|_{t=0} R_{\mu(t) \otimes \varepsilon_{0}} f$ and $\pm P f=\left.\frac{d^{+}}{d t}\right|_{t=0} R_{\varepsilon_{e} \otimes \varepsilon_{ \pm t}} . \quad W$ and $\pm P-$ by construction dissipative invariant operators - are extended to generators of continuous convolution semigroups $\left(\sigma_{t}:=\mu_{t} \otimes \varepsilon_{0}\right)_{t>0}$ and $\left(p_{t}^{ \pm}:=\varepsilon_{e} \otimes \varepsilon_{ \pm t}\right)_{t>0}$ respectively. (Cf. Theorem 2.3). Therefore, the steps in Section 2 yield the following result (cf. e.g., [14]), 2.14 III, [16], Theorem C. See also e.g., [15, 3] for applications:

Theorem 3.1. Let $\mathbb{G}$ be a locally compact group and $\mathbb{T}:=\left(T_{t}\right)_{t>0} \subseteq$ $\operatorname{Aut}(\mathbb{G})$ a fixed continuous one-parameter group. Furthermore, let $\Gamma:=$ $\mathbb{G} \rtimes \mathbb{R}$ denote the semidirect extension of $\mathbb{G}$ defined by $\mathbb{T}$. Then

a) $\quad \mathbb{D}:=\mathcal{D}(\mathbb{G}) \otimes \mathcal{D}(\mathbb{R})$ is a core for any continuous convolution semigroup of probabilities in $M_{*}^{1}(\Gamma)$.

b) There exists a bijection $(\mu(t))_{t \geq 0} \leftrightarrow\left(\mu_{t}\right)_{t>0}$ between $M$-semigroups and continuous convolution semigroups, i.e., between (distributions of) Ornstein-Uhlenbeck processes and (background driving) Lévy processes. The bijection is expressed by the 'forward and backward Lie-Trotter formulas'

$$
\mu(t)=\lim _{n \rightarrow \infty} \underset{k=0}{\star n-1} \underset{k t / n}{\star}\left(\mu_{t / n}\right)
$$

$$
\mu_{t}=\lim _{n \rightarrow \infty}(\mu(t / n))^{n}
$$

For (matrix cone-) hypergroups we shall prove in analogy to the group case:

Theorem 3.2. Let $\mathcal{K}$ be a matrix cone hypergroup (investigated in $[36$, 40]) with fixed continuous one parameter group $\mathbb{T}:=\left(T_{t}\right)_{t \geq 0} \subseteq \operatorname{Aut}(\mathcal{K})$. Define the semidirect hypergroup-product $\Gamma:=\mathbb{G} \rtimes \mathbb{R}$ in canonical way.

Then the assertions a) and b) of Theorem 3.1 hold true in this situation, where $\mathcal{D}(\mathbb{G})$ and $\mathbb{D}$ have to be replaced by suitable function spaces $\mathcal{A}$ and $\widetilde{\mathbb{D}}$ (defined in the proof of Theorem 4.21 and in 4.23 below) on the hypergroups $\mathcal{K}$ and $\Gamma$ respectively. 
In particular, $\widetilde{\mathbb{D}}$ is again a common core for all continuous convolution semigroups in $\mathcal{M}_{*}^{1}(\Gamma)$.

The proof of Theorem 3.1, worked out in Section 2, relied mainly on the Theorem $1.9 \mathrm{~b}$ ). In fact, Theorem 3.1, in particular a), is wellknown and was used several times - at least in the case of Lie groups without pointing out that the original version of Theorem 1.9 a) needs a straight forward generalization (i.e. condition (ii') instead of (ii)) to handle the case of semidirect products. (See e.g. [14], §2.14, [16]). We included a proof in order to show the differences to the case of hypergroups:

The proof of Theorem 3.2 is more complicated and not straight forward. In fact, the details are quite technical, but I was unable to find a better way. The proof will be carried out in Section 4, in a series of propositions, which may be interesting in their own right. Here we sketch an outline of the proof:

1. Assume $(\mu(t))_{t>0}$ to be a M-semigroup on $\mathcal{K}$ with corresponding space-time semigroup $\left(\lambda_{t}\right)$ in $\mathcal{M}_{*}^{1}(\Gamma)$. Then we construct a suitable core $\mathcal{E}$ for $\left(\lambda_{t}\right)$ such that on $\mathcal{E}$ the generator $U$ of the convolution operators $\left(R_{\lambda_{t}}\right)$ splits $U=W+P, W$ generating a continuous convolution semigroup $\left(\sigma_{t}=\mu_{t} \otimes \varepsilon_{0}\right)_{t \geq 0}$ concentrated on $\mathcal{K} \otimes\{0\} \cong \mathcal{K}$, and $P$ generates the semigroup of shifts $\left(p_{t}^{+}:=\varepsilon_{(e, t)}\right)_{t \geq 0}$. (Note that the constructed core $\mathcal{E}$ still depends on $\left(\lambda_{t}\right)$.)

2. Then the Lie-Trotter formula $(L T)$ (Proposition 2.4 a)) applied to $U=W+P$ yields $(L T 1)$. Hence $(\mu(t))_{t \geq 0} \mapsto\left(\mu_{t}\right)_{t \geq 0}$ is established.

3. Conversely, let $\left(\mu_{t}\right)$ be a continuous convolution semigroup on a matrix cone hypergroup $\mathcal{K}$. On these hypergroups there exists a subspace $\mathcal{A}$ which is a common core for all continuous convolution semigroups on $\mathcal{K}$ and is invariant under shifts and automorphisms. (Cf. $1.11,1.12)$. By means of $\mathcal{A}$ we construct a subspace $\widetilde{\mathbb{D}} \subseteq C_{0}(\Gamma)$ which is a common core for continuous convolution semigroups in $\mathcal{M}_{*}^{1}(\Gamma)$.

4. Furthermore, let $V$ be the generator of $\left(\mu_{t}\right)_{t>0}$, let $\left(\sigma_{t}:=\mu_{t} \otimes \varepsilon_{0}\right)_{t>0}$ with generator $W$, and let $P$ as above, then $\bar{U}=W+P$ is (the restriction to $\widetilde{\mathbb{D}}$ of) the generator of a continuous convolution semigroup $\left(\lambda_{t}=\mu(t) \otimes \varepsilon_{t}\right)_{t>0} \subseteq \mathcal{M}_{*}^{1}(\Gamma)$. Applying the Lie-Trotter formulas to $U=W+P$ resp. $W=U-P$ and considering the space component, i.e., the projection to $\mathcal{K}$, we obtain $(L T 1)$ and (LT2) respectively.

5. Together with step 1 . this yields the bijection $(\mu(t))_{t \geq 0} \leftrightarrow\left(\mu_{t}\right)_{t \geq 0}$ as asserted.

\section{Semidirect products $\Gamma=\mathcal{K} \rtimes \mathbb{R}$ : The Case of matrix CONE HYPERGROUPS $\mathcal{K}$}

As announced in Theorem 3.2 our aim is to establish a 1-1-correspondence between M-semigroups and continuous convolution semigroups on a class of hypergroups with 'group-like behaviour': Such hypergroups on the cone of non-negative definite matrices were recently investigated, cf. [36, 40], a class of hypergroups which share many features with locally compact groups. In particular, the group of automorphisms is well known, and there exist continuous one-parameter groups of automorphisms in abundance. (See e.g. [17] for an overview of some probabilistic structures on these hypergroups, in particular, 
the first section contains a collection of basic properties.) In the sequel we have these examples in mind, but results and proofs depend only on particular properties of $\mathcal{K}$, thus could be generalized to larger classes of hypergroups.

Definition 4.1. Let $\mathcal{K}$ be the cone of positive semidefinite $d \times d$ matrices endowed with a hypergroup structure (investigated in $[36,40]$ ). (We restrict for convenience to the case of real matrices.) $\mathcal{K}$ is a commutative Hermitean hypergroup, furthermore, self-dual (i.e., $\widehat{\mathcal{K}}$ is a hypergroup $\cong \mathcal{K})$, with Pontryagin and Godement property. In particular, Lévy's continuity theorem is valid. $\mathcal{K}$ is aperiodic, i.e., without idempotents except the unit e. The unit of the hypergroup $\mathcal{K}$ is the zero-matrix, denoted by e.

Automorphisms of $\mathcal{K}$ are obtained in the following way: $\mathcal{K}$ is considered as subset of the $d \times(d-1) / 2$-dimensional vector space $\mathbb{H}:=$ $\mathcal{K}-\mathcal{K}$ of (real) Hermitean matrices. For $a \in \mathrm{GL}\left(\mathbb{R}^{d}\right)$ put $T_{a}: \mathbb{H} \ni$ $\kappa \mapsto\left((a \kappa)(a \kappa)^{*}\right)^{1 / 2}=\left(a \kappa^{2} a^{*}\right)^{1 / 2} \in \mathcal{K}$. The restriction to $\mathcal{K}$ defines an hypergroup automorphism of $\mathcal{K}$. Let $\left(T_{t}\right)_{t \in \mathbb{R}}$ be a continuous one-parameter group in $\operatorname{Aut}(\mathcal{K})$. Then there exists a continuous one-parameter group $\left(a_{t}=\exp (t Q)\right)_{t \in \mathbb{R}} \subseteq \mathrm{GL}\left(\mathbb{R}^{d}\right)$ such that $T_{t}=T_{a_{t}}$ $\forall t \in \mathbb{R}$. And conversely, $\left(T_{a_{t}}\right) \subseteq \operatorname{Aut}(\mathcal{K})$ for any one-parameter group $\left(a_{t}\right)$. In the following we fix $T_{t}:=T_{a_{t}}$ with $a_{t}=\exp t \cdot Q, t \in \mathbb{R}$.

Let $\mathbb{V}:=\mathbb{H} \otimes \mathbb{R}$, the Cartesian product, containing $\Gamma:=\mathcal{K} \otimes \mathbb{R}$ as a subset. $\Gamma$, endowed with a convolution structure $\varepsilon_{(x, s)} * \varepsilon_{(y, t)}:=$ $\left(\varepsilon_{x} \star \varepsilon_{T_{s}(y)}\right) \otimes \varepsilon_{s+t}$ for $(x, s),(y, t) \in \Gamma$ and with involution defined by $(x, s)^{-}=\left(T_{-s}(x)^{-},-s\right)$ is a (non commutative) hypergroup. (The axioms are easily verified. Note that in our case, $\mathcal{K}$ is Hermitean, hence in particular, $T_{-s}(x)^{-}=T_{-s}(x)$.) Therefore, the notation $\Gamma=: \mathcal{K} \rtimes \mathbb{R}$ is justified.

Probabilities on $\mathcal{K}$ resp. on $\Gamma$ act by convolution on $C_{0}(\mathcal{K})$ and $C_{0}(\Gamma)$ respectively. We denote the left and right convolution operators as follows: Let $f \in C_{0}(\mathcal{K}), g \in C_{0}(\Gamma), z \in \mathcal{K},(z, r) \in \Gamma$.

$$
\begin{aligned}
\dot{R}_{z} f(x) & =f(x \star z)=\int_{\mathcal{K}} f(y) d\left(\varepsilon_{x} \star \varepsilon_{z}\right)(y) \\
\dot{L}_{z} f(x) & =f(z \star x)=\int_{\mathcal{K}} f(y) d\left(\varepsilon_{z} \star \varepsilon_{x}\right)(y) \\
R_{(z, r)} g(x, s) & =f((x, s) *(z, r))=\int_{\Gamma} g(y, u) d\left(\varepsilon_{(x, s)} * \varepsilon_{(z, r)}\right)(y, u) \\
L_{(z, r)} g(x, s) & =f((z, r) *(x, s))=\int_{\Gamma} g(y, u) d\left(\varepsilon_{(z, r)} * \varepsilon_{(x, s)}\right)(y, u)
\end{aligned}
$$

In an analogous way we define for measures $\lambda$ on $\Gamma$ resp. $\mu$ on $\mathcal{K}$ the left resp. right convolution operators $\dot{R}_{\mu}, \dot{L}_{\mu}, R_{\lambda}, L_{\lambda}$ on $\mathcal{K}$ resp. $\Gamma$.

Definition 4.2. In the following we restrict again our considerations to measures on the 'space-time building' $\Gamma$ of the particular form $\lambda=$ $\mu \otimes \varepsilon_{u} \in \mathcal{M}_{*}^{1}(\Gamma):=\left\{\mu \otimes \varepsilon_{u}: \mu \in \mathcal{M}^{1}(\mathcal{K}), u \in \mathbb{R}\right\}$. In that case we have

$$
\begin{aligned}
& R_{\mu \otimes \varepsilon_{u}} g(x, s)=\int_{\mathcal{K}} g\left(x \star T_{s}(y), s+u\right) d \mu(y) \\
& L_{\mu \otimes \varepsilon_{u}} g(x, s)=\int_{\mathcal{K}} g\left(y \star T_{u}(x), s+u\right) d \mu(y)
\end{aligned}
$$


Note that for $g=\varphi \otimes \psi$ we obtain (with $\psi_{u}: s \mapsto \psi(s+u)$ ):

$$
\begin{aligned}
& R_{\mu \otimes \varepsilon_{u}} g(x, s)=\int_{\mathcal{K}} \varphi\left(x \star T_{s}(y)\right) d \mu(y) \cdot \psi_{u}(s)=\left(\dot{R}_{T_{s}(\mu)} \varphi\right)(x) \cdot \psi_{u}(s) \\
& L_{\mu \otimes \varepsilon_{u}} g(x, s)=\int_{\mathcal{K}} \varphi\left(y \star T_{u}(x)\right) d \mu(y) \cdot \psi_{u}(s)=\left(\dot{L}_{\mu} \varphi\right)\left(T_{u}(x)\right) \cdot \psi_{u}(s)
\end{aligned}
$$

The involution on $\Gamma$ induces involutions on on spaces of functions and measures:

Let $g \in \mathbb{C}^{b}(\Gamma)$. Then $\widetilde{g}(x, s):=g\left((x, s)^{-}\right)=g\left(T_{-s}(x),-s\right)$

Let $\lambda \in \mathcal{M}^{b}(\Gamma)$. Then $\int_{\Gamma} f d \widetilde{\lambda}:=\int_{\Gamma} \tilde{f} d \lambda$

In particular, for $\lambda=\mu \otimes \varepsilon_{u}$ we obtain $\tilde{\lambda}=T_{-u}(\mu) \otimes \varepsilon_{-u}$.

We recall the notations of left invariant operators and subspaces introduced in Section 2; we have to distinguish between invariant operators on $\mathcal{K}$ and on the non-commutative hypergroup $\Gamma$.

Proposition 4.3. a) For $\lambda, \mu \in \mathcal{M}^{b}(\Gamma)$ we have $\widetilde{(\lambda * \mu)}=\widetilde{\mu} * \widetilde{\lambda}$

b) For $\lambda \in \mathcal{M}^{b}(\Gamma), f \in C_{0}(\Gamma)$ we have $\widetilde{\left(R_{\lambda} f\right)}=L_{\tilde{\lambda}} \tilde{f}$

The existence of background driving Lévy processes: the mapping $(\mu(t))_{t \geq 0} \mapsto\left(\mu_{t}\right)_{t \geq 0}$.

The hypergroup $\mathcal{K}$ is embedded into a vector space $\mathbb{H}$, hence inherits a differentiable structure. Note that the action of $T_{t}$ on $\mathcal{K}$ resp. $\mathbb{H}$ is smooth: $t \mapsto\left(T_{\exp t Q}(\kappa)\right)^{2}=\exp t Q \kappa^{2} \exp t Q^{*}=: \kappa(t)^{2}$ is an entire function, and $\mathcal{K} \ni x \mapsto x^{1 / 2} \in \mathcal{K}$ is holomorphic on $\mathcal{K}_{0}:=$ $\mathcal{K} \cap \mathrm{GL}\left(\mathbb{R}^{d}\right)$. If the kernel $N(\kappa) \neq\{0\}$ then $N(\kappa(t))=\exp \left(-t Q^{*}\right) N(\kappa)$ and $N(\kappa(t))^{\perp}=\exp (-t Q) N(\kappa)^{\perp}$, hence the projections onto these subspaces depend analytically on $t$. Whence the assertion easily follows.

We define particular differential operators:

Definition 4.4. For $f \in C_{0}^{(1)}(\mathbb{H} \otimes \mathbb{R})$ (i.e. with continuous derivatives in $C_{0}(\mathbb{H} \otimes \mathbb{R})$ ) and $(x, s) \in \mathbb{H} \otimes \mathbb{R}$ we put

$$
\begin{aligned}
X f(x, s) & :=\left.\frac{d^{+}}{d t}\right|_{t=0} f\left(T_{t}(x), s+t\right)=\lim _{t \searrow 0} \frac{1}{t}\left(f\left(T_{t}(x), s+t\right)-f(x, s)\right) \\
\operatorname{Pf}(x, s) & :=\left.\frac{d^{+}}{d t}\right|_{t=0} f(x, s+t)=\lim _{t \searrow 0} \frac{1}{t}(f(x, s+t)-f(x, s)) \\
S f(x, s) & :=\left.\frac{d^{+}}{d t}\right|_{t=0} f\left(T_{t}(x), s\right)=\lim _{t \searrow 0} \frac{1}{t}\left(f\left(T_{t}(x), s\right)-f(x, s)\right)
\end{aligned}
$$

For the restriction to $(x, s) \in \Gamma$ we obtain:

Proposition 4.5. Let $\lambda \in \mathcal{M}^{b}(\Gamma), f \in C_{0}^{(1)}(\mathbb{H} \otimes \mathbb{R}),(x, s) \in \Gamma$

a) $X f(x, s)=\lim _{t \searrow 0} L_{\frac{1}{t}\left(\varepsilon_{(e, t)}-\varepsilon_{(e, 0)}\right)} f(x, s)$

b) $\quad P f(x, s)=\lim _{t \backslash 0} R_{\frac{1}{t}\left(\varepsilon_{(e, t)}-\varepsilon_{(e, 0)}\right)} f(x, s)$

Hence

$$
\begin{array}{ll}
\text { c) } & R_{\lambda} X f(x, s)=X R_{\lambda} f(x, s) \quad \text { d) } \quad L_{\lambda} P f(x, s)=P L_{\lambda} f(x, s) \\
\text { e) } & \sup _{(x, s) \in \Gamma}\left|X R_{\lambda} f(x, s)\right| \leq\|\lambda\| \sup _{(x, s) \in \Gamma}|X f(x, s)| \\
\text { f) } & \sup _{(x, s) \in \Gamma}\left|S R_{\lambda} f(x, s)\right| \leq\|\lambda\| \sup _{(x, s) \in \Gamma} \mid S f((x, s) \mid .
\end{array}
$$


( a)-e) are obvious, only f) needs a proof:

It is sufficient to prove the assertion for $\lambda=\varepsilon_{(y, u)}$. A simple calculation shows $S R_{(y, u)} f(x, s)=R_{\left(T_{s}(y), u\right)} S f(x, s)$. Whence

$$
\begin{aligned}
& \sup _{(x, s) \in \Gamma}\left|S R_{(y, u)} f(x, s)\right|=\sup _{(x, s) \in \Gamma}\left|R_{\left(T_{s}(y), u\right)} S f(x, s)\right| \leq \\
& \sup _{\left(y^{\prime}, u\right) \in \Gamma} \sup _{(x, s) \in \Gamma}\left|R_{\left(y^{\prime}, u\right)} S f(x, s)\right| \leq \sup _{\left(y^{\prime}, u\right) \in \Gamma}\left\|R_{\left(y^{\prime}, u\right)} S f\right\|_{C_{0}(\Gamma)} \leq\|S f\|_{C_{0}(\Gamma) .} \|
\end{aligned}
$$

Proposition 4.6. Let $f \in C_{0}^{(1)}(\mathbb{H} \otimes \mathbb{R}),(x, s) \in \mathbb{H} \otimes \mathbb{R}$.

$$
\begin{aligned}
& X f(x, s)=\lim _{t \searrow 0} \frac{1}{t}\left(f\left(T_{t}(x), s\right)-f(x, s)\right)+\lim _{t \searrow 0} \frac{1}{t}(f(x, s+t)-f(x, s)) \\
& =: S f(x, s)+\operatorname{Pf}(x, s) \\
& \llbracket X f(x, s)= \\
& \lim _{t \searrow 0}\left[\frac{1}{t}\left(f\left(T_{t}(x), s+t\right)-f(x, s+t)\right)+\frac{1}{t}(f(x, s+t)-f(x, s))\right]
\end{aligned}
$$

The second terms converge to $\operatorname{Pf}(x, s)$, hence also the first terms are convergent, to $S^{\prime} f(x, s)$ say. Now

$$
\begin{aligned}
& S^{\prime} f(x, s)=\lim _{t \searrow 0}\left[\frac{1}{t}\left(f\left(T_{t}(x), s+t\right)-f\left(T_{t}(x), s\right)\right)\right. \\
& \left.\left.+\frac{1}{t}\left(f\left(T_{t}(x), s\right)-f(x, s)\right)-\frac{1}{t}(f(x), s+t)-f(x, s)\right)\right]
\end{aligned}
$$

The first and third terms converge to $\operatorname{Pf}(x, s)$ and $-\operatorname{Pf}(x, s)$ respectively, hence $S^{\prime} f=S f$ as asserted.

The differential operators $X$ and $P$ are related by

Proposition 4.7. $(X \widetilde{f})(x, s)=-(\widetilde{P f})(x, s)$

$$
\begin{aligned}
{[X \widetilde{f}(x, s)} & =\lim _{t \searrow 0} \frac{1}{t}\left(\tilde{f}\left(T_{t}(x), s+t\right)-\tilde{f}(x, s)\right) \\
& =\lim _{t \searrow 0} \frac{1}{t}\left(f\left(T_{-s-t} T_{t}(x),-s-t\right)-f\left(T_{-s}(x),-s\right)\right) \\
& =\lim _{t \searrow 0} \frac{1}{t}\left(f\left(T_{s}(x),-s-t\right)-f\left(T_{-s}(x),-s\right)\right) \\
& =-(P f)\left(T_{-s}(x),-s\right)=-(\widetilde{P f})(x, s)
\end{aligned}
$$

Definition 4.8. We introduce semi-norms on $C_{0}^{(1)}(\mathbb{H} \otimes \mathbb{R})$ :

$$
\|f\|_{(0)}:=\sup _{(x, s) \in \Gamma}|f(x, s)|, \quad\|f\|_{(1)}:=\sup _{(x, s) \in \Gamma}|X f(x, s)|=\|X f\|_{(0)}
$$
and $\|f\|_{(2)}:=\|S f\|_{(0)}$. Finally we put $\|\mid f\|\left\|:=\sum_{j=0}^{2}\right\| f \|_{(j)}$.

$\mathcal{B}$ denotes the completion of $C_{0}^{(1)}(\mathbb{H} \otimes \mathbb{R})$ w.r.t. ||$|\cdot|||$. (Since functions coinciding on $\Gamma$ are identified, the Banach space $\mathcal{B}$ may be considered as subspace of $C_{0}(\Gamma)$.)

Proposition 4.9. a) $\mathcal{B}$ is dense in $C_{0}(\Gamma)$ w.r.t. $\|\cdot\|_{\infty}\left(=\|\cdot\|_{(0)}\right)$.

b) For all $f \in \mathcal{B}$ there exist $X f, P f, S f \in C_{0}(\Gamma)$.

c) For all $\lambda \in \mathcal{M}^{b}(\Gamma)$, for all $f \in \mathcal{B}$ we have $\left\|\left|R_{\lambda} f\||\leq\|\lambda\| \cdot\|\mid\| f\|\|\right.\right.$.

d) In particular, for a continuous convolution semigroup $\left(\lambda_{t}\right)_{t \geq 0}$ in $\mathcal{M}^{(1)}(\Gamma)$ the operators $\left(R_{\lambda_{t}}\right)_{t \geq 0}$ may be considered as $C_{0}-$ contraction semigroup on $C_{0}(\Gamma)$ as well as on $\mathcal{B}$. 
[ a), b) are obvious, c) and d) are immediate consequences of Proposition $4.5 \mathrm{e}$ ) and $\mathrm{f})$. \

Definition 4.10. In the following let $\left(\lambda_{t}=\mu(t) \otimes \varepsilon_{t}\right)_{t \geq 0}$ be a continuous convolution semigroup in $\mathcal{M}_{*}^{1}(\Gamma)$ with $\lambda_{0}=\varepsilon_{(e, 0)}$. Let $(U, D(U))$ resp. $(\stackrel{*}{U}, D(\stackrel{*}{U}))$ denote the infinitesimal generators of the $C_{0}-$ contraction semigroups $\left(R_{\lambda_{t}}\right)_{t \geq 0}$ on $C_{0}(\Gamma)$ and on $\mathcal{B}$ respectively.

Proposition 4.11. $D(\stackrel{*}{U})$ is dense in $D(U)$ and in $C_{0}(\Gamma)$, furthermore, $D(\stackrel{*}{U})$ is a core for $(U, D(U))$.

II In fact, by construction $D(\stackrel{*}{U}) \subseteq D(U)$ and $D(\stackrel{*}{U})$ is dense in $\mathcal{B}$ w.r.t. $\|\mid \cdot\|$. Hence also dense in $C_{0}(\Gamma)$ w.r.t. $\|\cdot\|_{(0)}$. Furthermore, $(I-\stackrel{*}{U}) D(\stackrel{*}{U})=\mathcal{B}$, hence $(I-U) D(\stackrel{*}{U})$ is dense in $C_{0}(\Gamma)$. Whence the assertion.

Remark 4.12. $D(U)$ is left invariant since $U$ is left invariant. But the ||$|\cdot| \mid-$-defining operators $X$ and $S$ are not left invariant. Hence we can not conclude that $D(\stackrel{*}{U})$ is left invariant. That is the reason why we have to use more complicated constructions in the sequel

Proposition 4.13. There exists a core $\mathcal{E}$ for $\left(R_{\lambda_{t}}\right)_{t \geq 0}($ resp. $(U, D(U)))$ such that $\mathcal{E} \subseteq D(U) \cap D(P)$

Proof: Let $f \in D(U), \psi \in \mathcal{D}(\mathbb{R})$. Put $g=g_{f, \psi}:(x, s) \mapsto f(x, s) \cdot \psi(s)$.

Let $\mathcal{E}_{0}:=\operatorname{span}\left\{g_{f, \psi}: f \in D(U), \psi \in \mathcal{D}(\mathbb{R})\right\}$.

1. $\mathcal{E}_{0} \subseteq D(U)$.

In fact, we prove for $g:=g_{f, \psi}: U g(x, s)=U f(x, s) \cdot \psi(s)+f(x, s) \cdot \psi^{\prime}(s)$ :

$$
\begin{aligned}
\mathbb{} & \frac{1}{t} \int_{\mathcal{K}} g\left(x \star T_{s}(y), s+t\right)-g(x, s) d \mu(t)(y) \\
= & \frac{1}{t} \int_{\mathcal{K}} f\left(x \star T_{s}(y), s+t\right) \cdot \psi(s+t)-f(x, s) \cdot \psi(s) d \mu(t)(y) \\
= & {\left[\frac{1}{t} \int_{\mathcal{K}} f\left(x \star T_{s}(y), s+t\right)-f(x, s) d \mu(t)(y)\right] \cdot \psi(s+t) } \\
+ & \int_{\mathcal{K}} f(x, s) d \mu(t)(y) \cdot\left[\frac{1}{t}(\psi(s+t)-\psi(s))\right] \\
\stackrel{t \rightarrow 0}{\longrightarrow} & U f(x, s) \cdot \psi(s)+\psi^{\prime}(s) \cdot f(x, s) .
\end{aligned}
$$

Convergence is uniform in $(x, s)$ since $\psi$ and $\psi^{\prime}$ have compact support and $U f \in C_{0}(\Gamma)$.

2. $\mathcal{E}_{0}$ is dense in $C_{0}(\Gamma)$. In fact, let $L_{n}$ be compact intervals, $L_{n} \nearrow \mathbb{R}$, e.g., $L_{n}=\left[-k_{n}, k_{n}\right]$ with $k_{n} \nearrow \infty$. Let $\psi_{n} \in \mathcal{D}(\mathbb{R}), 1_{L_{n}} \leq \psi_{n} \leq 1_{L_{n+1}}$. Then $f(x, s) \cdot \psi_{n}(s) \rightarrow f(x, s)$ uniformly in $(x, s) \in \Gamma$ (since $\left.f \in C_{0}(\Gamma)\right)$.

3. $(I-U) \mathcal{E}_{0}$ is dense in $C_{0}(\Gamma)$.

【 We show: $\forall \varepsilon>0 \forall f \in D(U)$ there exists a $g \in \mathcal{E}_{0}$ such that $\|(I-U) f-(I-U) g\|_{\infty}=\|(f-g)-(U f-U g)\|_{\infty}<\varepsilon$. (Note that $(I-U) D(U)=C_{0}(\mathcal{K})$.)

Let $f \in D(U)$, choose $L_{n}, \psi_{n}$ as above, and assume in addition that $\left\|\psi_{n}{ }^{\prime}\right\|_{\infty} \rightarrow 0$. Put $g_{n}(x, s):=f(x, s) \cdot \psi_{n}(s)$. Then $(I-U) g_{n}(x, s)=$ $g_{n}(x, s)-U f(x, s) \cdot \psi_{n}(s)-f(x, s) \cdot \psi_{n}^{\prime}(s)$, therefore, $\quad \mid(I-U) f(x, s)-$ 
$(I-U) g_{n}(x, s)\left|\leq\left\|f-g_{n}\right\|_{(0)}+\right| U f(x, s)|\cdot| 1-\psi_{n}(s) \mid+\|f\|_{(0)} \cdot\left\|\psi_{n}^{\prime}\right\|_{\infty} \rightarrow$

0 . Convergence is again uniform in $(x, s)$ since $U f \in C_{0}(\Gamma)$. ]

4. The above steps remain true if $\mathcal{E}_{0}$ is replaced by

$$
\mathcal{E}:=\operatorname{span}\left\{g_{f, \psi}: f \in D(\stackrel{*}{U}), \psi \in \mathcal{D}(\mathbb{R})\right\} .
$$

(According to $4.11, D(\stackrel{*}{U})$ is a core for $(U, D(U))$.)

5. In that case we have in addition $\mathcal{E} \subseteq D(P)$ (and $P \mathcal{E} \subseteq C_{0}(\Gamma)$ ).

【 Since $D(\stackrel{*}{U}) \subseteq \mathcal{B} \subseteq D(P)$ (cf. Proposition 4.9) and $P g_{f, \psi}(x, s)=$ $\left.\left.P f(x, s) \cdot \psi(s)+f(x, s) \cdot \psi^{\prime}(s).\right)\right]$

Note that in contrast to $\mathcal{E}_{0}$ the core $\mathcal{E}$ is not left invariant but the core $D(U) \cap D(P)$ is:

Proposition 4.14. $D(U) \cap D(P)$ is a core for $(U, D(U))$ (since the core $\mathcal{E}$ is contained in $D(U) \cap D(P)$ according to 4.13). Furthermore, $D(U) \cap$ $D(P)$ is obviously left invariant, since $U$ and $P$ are left invariant.

For $f \in D(U) \cap D(P)$ we have:

$$
U f=W f+P f,
$$

where $W f(x, s)=\lim _{t \searrow 0} \frac{1}{t} \int_{\mathcal{K}} f\left(x \star T_{s}(y), s\right)-f(x, s) d \mu(t)(y)$

$=\lim _{t \searrow 0} \frac{1}{t}\left(R_{\mu(t) \otimes \varepsilon_{0}}-I\right) f(x, s)$

$\llbracket U f(x)=\lim _{t \searrow 0} \frac{1}{t} \int_{\mathcal{K}} f\left(x \star T_{s}(y), s+t\right)-f(x, s) d \mu(t)(y)=$

$=\lim _{t \searrow 0} \frac{1}{t} \int_{\mathcal{K}} f\left(x \star T_{s}(y), s+t\right)-f(x, s+t) d \mu(t)(y)+$

$\lim _{t \searrow 0} \frac{1}{t} \int_{\mathcal{K}} f(x, s+t)-f(x, s) d \mu(t)(y)=: W f(x, s)+\operatorname{Pf}(x, s)$

Furthermore,

$$
\begin{aligned}
& W f(x, s)=\lim _{t \searrow 0}\left[\frac{1}{t} \int_{\mathcal{K}} f\left(x \star T_{s}(y), s\right)-f(x, s) d \mu(t)(y)\right. \\
& +\frac{1}{t} \int_{\mathcal{K}} f\left(x \star T_{s}(y), s+t\right)-f\left(x \star T_{s}(y), s\right) d \mu(t)(y) \\
& \left.-\frac{1}{t} \int_{\mathcal{K}} f(x, s+t)-f(x, s) d \mu(t)(y)\right]
\end{aligned}
$$

The second and the third terms converge to $\operatorname{Pf}(x, s)$ and $-P f(x, s)$ respectively, whence

$$
W f(x, s)=\lim _{t \searrow 0} \frac{1}{t} \int_{\mathcal{K}} f\left(x \star T_{s}(y), s\right)-f(x, s) d \mu(t)(y)
$$

follows.

Definition 4.15. $\Lambda:=\{\mathcal{K} \ni x \mapsto f(x, 0)=: \dot{f}(x): f \in D(U) \cap D(P)\}$

Proposition 4.16. $\Lambda$ is $\|\cdot\|$-dense in $C_{0}(\mathcal{K})$, left invariant (and also right invariant, as $\mathcal{K}$ is Abelian).

【 $D(U) \cap D(P)$ is a left invariant subspace of $C_{0}(\Gamma)$. In other words, $L_{(y, u)}(D(U) \cap D(P)) \subseteq(D(U) \cap D(P)) \forall(y, u) \in \Gamma$. Considering $u=0$ we obtain $\dot{L}_{y}(\Lambda) \subseteq \Lambda \forall y \in \mathcal{K}$. 
Now we are ready to prove the existence of a background driving Lévy process:

Proposition 4.17. As introduced afore, we put $\dot{f}$ for the restriction of $f$ to $\{(y, 0): y \in \mathcal{K}\} \equiv \mathcal{K}$. With this notation we have:

$$
\Lambda \ni \dot{f} \mapsto W f(\cdot, 0)=: V \dot{f}
$$

is a left invariant operator $\Lambda \rightarrow C_{0}(\mathcal{K})$. $V$ is dissipative (by construction) and has a unique extension to the generator of a semigroup of convolution operators $\left(R_{\mu_{t}}\right)_{t \geq 0}$ for a continuous convolution semigroup $\left(\mu_{t}\right)_{t \geq 0} \subseteq \mathcal{M}_{+}^{(1)}(\mathcal{K})$. In particular, $\Lambda$ is a core for $\left(\mu_{t}\right)_{t \geq 0}$.

I $\Lambda$ is dense in $C_{0}(\mathcal{K})$ and left invariant. Since $\mathcal{K}$ is Abelian, $\Lambda$ is (trivially) right invariant. By construction, $V$ is dissipative, whence according to Theorem 1.9 a) the existence of $\left(\mu_{t}\right)_{t \geq 0} \subseteq \mathcal{M}^{(1)}(\mathcal{K})$ follows.

Furthermore, according to Proposition 4.14,V $=\lim _{t \backslash 0} V_{t}$ where $V_{t}=$ $\frac{1}{t}\left(R_{\mu(t)}-I\right)$ and $\mu(t) \in \mathcal{M}^{1}(\mathcal{K})$. Hence $R_{\mu_{s}}=\lim _{t \searrow 0} \exp s \cdot V_{t}$, thus $\mu_{s}=\lim _{t \searrow 0} \exp s \frac{1}{t}\left(\mu(t)-\varepsilon_{e}\right) \geq 0$ for all $s \geq 0$.

Proposition 4.18. Let $\left(\mu_{t}\right)_{t \geq 0} \subseteq \mathcal{M}_{+}^{(1)}(\mathcal{K}), W$ and $V$ be defined as in Proposition 4.17. Let $\left(\sigma_{t}:=\mu_{t} \otimes \varepsilon_{0}\right)_{t \geq 0} \subseteq \mathcal{M}_{*}^{1}(\Gamma)$ denote the corresponding continuous convolution semigroup, concentrated on $\mathcal{K} \otimes\{0\} \cong$ $\mathcal{K}$. Put furthermore $\left(p_{t}^{ \pm}:=\varepsilon_{(e, \pm t)}\right)_{t \in \mathbb{R}_{+}}$.

Then $W$ and $\pm P$ are the generators of $\left(R_{\sigma_{t}}\right)_{t \geq 0}$ and $\left(R_{p_{t}^{ \pm}}\right)_{t \geq 0}$ respectively.

〔 For all $(x, s) \in \Gamma$ we have:

$$
\begin{aligned}
& W f(x, s)=\lim _{t \searrow 0} \frac{1}{t} \int_{\mathcal{K}} f\left(x \star T_{s}(y), s\right)-f(x, s) d \mu(t)(y) \\
& =\lim _{t \searrow 0} \frac{1}{t} \int_{\mathcal{K}}\left(L_{(e, s)} f\right)\left(T_{-s}(x) \star y, 0\right)-\left(L_{(x, s)} f\right)\left(T_{-s}(x), 0\right) d(\mu(t)(y) \\
& =V \dot{g}\left(T_{-s}(x)\right) \quad\left(\text { with } g:=L_{(e, s)} f\right) \\
& =\left.\frac{d^{+}}{d t}\right|_{t=0} \dot{R}_{\mu_{t}} \dot{g}\left(T_{-s}(x)\right)=\left.\frac{d^{+}}{d t}\right|_{t=0} R_{\sigma_{t}} g\left(T_{-s}(x), 0\right) \\
& =\left.\frac{d^{+}}{d t}\right|_{t=0} R_{\sigma_{t}} f(x, s)
\end{aligned}
$$

(cf. Proposition 4.17.)

In view of Proposition 4.14, application of the Lie-Trotter formula (LT) (Proposition 2.4 a)) to the decomposition $U=W+P$ yields

Proposition 4.19. With the notations introduced above we obtain:

$$
\mu(t)=\lim _{n \rightarrow \infty} \underset{k=0}{n-1} \underset{k t / n}{\star}\left(\mu_{t / n}\right)
$$

〔 The Lie-Trotter formula $(L T *)$ yields $\lambda_{t}=\lim _{n \rightarrow \infty}\left(\sigma_{t / n} \star p_{t / n}^{+}\right)^{n}$. Considering the projection to the $\mathcal{K}$-component yields (LT1). 
In 4.17 we have proved $\mu_{t} \in \mathcal{M}_{+}^{(1)}(\mathcal{K})$. Now we are ready to prove

Proposition 4.20. $\mu_{t} \in \mathcal{M}^{1}(\mathcal{K})$ for all $t \geq 0$.

I Assume $\left\|\mu_{t}\right\|<1$ for some $t>0$. Then, as $\mu_{t}$ are positive, $\left\|\mu_{t}\right\|=$ $e^{-c t}$ for some $c>0$. Therefore, in (LT1) the right hand side has norm $\leq e^{-c t}$. A contradiction to the assumption $\mu(t) \in \mathcal{M}^{1}(\mathcal{K})$.

We have proved that for any M-semigroup $(\mu(t))_{t \geq 0}$ there exists a continuous convolution semigroup $\left(\mu_{t}\right)_{t \geq 0}$, the background driving Lévy process, such that $(L T 1)$ holds true. In fact, the following results prove uniqueness of $\left(\mu_{t}\right)_{t>0}$ and bijectivity of the mapping $\left(\mu_{t}\right)_{t>0} \mapsto$ $(\mu(t))_{t \geq 0}$. Bijectivity is proved by the inverse Lie-Trotter formula $(\bar{L} T 2)$.

The existence of M-semigroups: The mapping $(\mu(t))_{t \geq 0} \mapsto$ $\left(\mu_{t}\right)_{t \geq 0}$

First we show

Theorem 4.21. Let $\left(\mu_{t}\right)_{t \geq 0}$ be a continuous convolution semigroup in $\mathcal{M}^{1}(\mathcal{K})$. Then there exists a M-semigroup $(\mu(t))_{t \geq 0} \subseteq \mathcal{M}^{1}(\mathcal{K})$ such that (LT1) and (LT2) hold:

$$
(L T 1) \quad \mu(t)=\lim _{n \rightarrow \infty} \underset{k=0}{\star n-1} \star T_{k t / n}\left(\mu_{t / n}\right) \quad(L T 2) \quad \mu_{t}=\lim _{n \rightarrow \infty}(\mu(t / n))^{n}
$$

Proof: At the first glance it seems obvious to consider as before

$$
W=\left.\frac{d^{+}}{d t}\right|_{t=0} R_{\mu_{t} \otimes \varepsilon_{0}}=:\left.\frac{d^{+}}{d t}\right|_{t=0} R_{\sigma_{t}}
$$

and to apply the Lie-Trotter formula to the representation $U=W+P$ resp. $W=U-P$. But a priori there is no 'natural' common domain for $U, W, P$. Therefore we have to find a slightly different approach. This will be done in the subsequent steps, formulated as propositions.

Let $\left(\mu_{t}\right)_{t \geq 0} \in \mathcal{M}^{1}(\mathcal{K})$ be given, define $\left(\sigma_{t}:=\mu_{t} \otimes \varepsilon_{0}\right)_{t \geq 0} \subseteq \mathcal{M}_{*}^{1}(\Gamma)$, put for $t>0, W_{t}:=\frac{1}{t}\left(R_{\sigma_{t}}-I\right), V_{t}:=\frac{1}{t}\left(\dot{R}_{\mu_{t}}-I\right)$ (acting on $C_{0}(\Gamma)$ and $C_{0}(\mathcal{K})$ respectively). Furthermore, let $(W, D(W))$ and $(V, D(V))$ be the generators of the corresponding contraction semigroups $\left(R_{\sigma_{t}}\right)$ and $\left(\dot{R}_{\mu_{t}}\right)$.

Let $\mathcal{A} \subseteq D(V)$ denote a core for $\left(\mu_{t}\right)_{t \geq 0}$ with the following properties:

(1) $\mathcal{A}$ is left invariant (and right invariant, as $\mathcal{K}$ is Abelian).

(2) $T_{s}(\mathcal{A}) \subseteq \mathcal{A}$ for all automorphisms $T_{s}$.

[ Such cores exist for $\mathcal{K}$, e.g., $\mathcal{A}=\left(L_{c}^{1}(\widehat{\mathcal{K}})\right)^{\vee}$, the space of analytic vectors, as mentioned in $1.11,1.12$. ]

Define $\mathbb{D}:=\mathcal{A} \otimes \mathcal{D}(\mathbb{R}) \subseteq C_{0}(\Gamma)$. Then we have:

(i) $\mathbb{D} \subseteq D(W)$

$\llbracket$ Let $f:=\varphi \otimes \psi \in \mathbb{D}$. Then

$W_{t} f(x, s)=\frac{1}{t}\left(\int_{\mathcal{K}} \varphi\left(x \star T_{s}(y)\right)-\varphi(x) d \mu_{t}(y)\right) \cdot \psi(s)$

$=\left(V_{t} \gamma\right)\left(T_{-s}(x)\right) \cdot \psi(s) \stackrel{t \rightarrow 0}{\longrightarrow}(V \gamma)\left(T_{-s}(x)\right) \cdot \psi(s)$

(with $\gamma:=\varphi \circ T_{s} \in \mathcal{A}$.) We define: $\left.W f(x, s):=\lim _{t \searrow 0} W_{t} f(x, s)\right]$

(ii) $\mathbb{D}$ is left invariant and dense in $C_{0}(\Gamma)$ 
【 Obviously $\mathbb{D}$ is dense in $C_{0}(\Gamma)$. To prove invariance we consider $L_{(y, t)}(\varphi \otimes \psi)(x, s)=(\varphi \otimes \psi)\left(y \star T_{t}(x), s+t\right)=$ $\left(\varphi \circ T_{t}\right)\left(T_{-t}(y) \star x\right) \cdot \psi(s+t)=\dot{L}_{\left(T_{-t}(y)\right)}\left(\varphi \circ T_{t}\right)(x) \cdot \psi(s+t)=: g(x) \cdot \xi(s)$ with $g=\dot{L}_{\left(T_{-t}(y)\right)}\left(\varphi \circ T_{t}\right) \in \mathcal{A}$ and $\xi \in \mathcal{D}(\mathbb{R})$. ]

Proposition 4.22. Let $f \in \mathbb{D},(z, u) \in \Gamma$. Then $R_{(z, u)} f \in D(W)$.

『In fact, by definition

$$
\begin{aligned}
& W_{t} R_{(z, u)}(\varphi \otimes \psi)(x, s)= \\
& =\frac{1}{t} \int\left(\varphi\left(x \star T_{s}(z) \star T_{s+u}(y)\right)-\varphi\left(x \star T_{s}(z)\right) d \mu_{t}(y) \cdot \psi(s+u)\right. \\
& =V_{t}\left(\varphi \circ T_{s+u}\right)\left(T_{-(s+u)}(x) \star T_{u}(z)\right) \cdot \psi(s+u) \\
& =: \dot{R}_{z}\left(\left(V_{t} \varphi_{s, u}\right) \circ T_{-u}\right)\left(T_{-s}(x)\right) \cdot \psi_{u}(s) \quad\left(\text { with } \varphi_{s, u}:=\varphi \circ T_{s+u}\right) \\
& \stackrel{t \rightarrow 0}{\longrightarrow} \dot{R}_{z}\left(\left(V \varphi_{s, u}\right) \circ T_{-u}\right)\left(T_{-s}(x)\right) \cdot \psi_{u}(s) \\
& =V\left(\varphi \circ T_{s+u}\right)\left(T_{-(s+u)}(x) \star T_{u}(z)\right) \cdot \psi(s+u) \\
& =: W\left(R_{(z, u)}(\varphi \otimes \psi)\right)(x, s)
\end{aligned}
$$

Convergence is again uniform on $\Gamma$.

Definition 4.23. Let $\widetilde{\mathbb{D}}:=\operatorname{span}\left\{R_{(z, u)} f:(z, u) \in \Gamma, f \in \mathbb{D}\right\}$

Proposition 4.24. $\widetilde{\mathbb{D}}$ is dense in $C_{0}(\Gamma)$ and left and right invariant.

Furthermore, $\widetilde{\mathbb{D}} \subseteq D(W) \cap D(P)$.

$W$ and $\pm P$ are, as limits of convolution operators, left invariant and by construction dissipative. Hence $U$ shares this property.

Therefore, according to Theorem $1.9 \mathrm{c}), \widetilde{\mathbb{D}}$ is a core for $P, W$ and $U:=W+P$. (Note that $W=U-P$.

【 Only $\widetilde{\mathbb{D}} \subseteq D(P)$ needs a proof:

$$
\begin{gathered}
P R_{(z, u)}(\varphi \otimes \psi)(x, s)=\lim _{t \searrow 0} R_{\frac{1}{t}\left(\varepsilon_{(e, t)}-\varepsilon_{(e, 0)}\right)} R_{(z, u)}(\varphi \otimes \psi)(x, s)= \\
\lim _{t \searrow 0}\left(\varphi\left(x \star T_{s}(z)\right)\right) \cdot \frac{1}{t}(\psi(s+u+t)-\psi(s+u))=\varphi\left(x \star T_{s}(z)\right) \cdot \psi^{\prime}(s+u)
\end{gathered}
$$

Convergence is uniform since $\psi$ and $\psi^{\prime}$ have compact supports. Whence the assertion.

Proposition 4.25. The afore announced Lie-Trotter formulas (LT1) and (LT2) (cf. 4.21) hold.

I Applying the Lie-Trotter formula $(L T)$ (cf. Proposition 2.4 a)) to $U=W+P$ resp. $W=U-P$ yields $\lambda_{t}=\lim _{n \rightarrow \infty}\left(\sigma_{t / n} * p_{t / n}^{+}\right)^{n}$ resp. $\sigma_{t}=\lim _{n \rightarrow \infty}\left(\lambda_{t / n} * p_{t / n}^{-}\right)^{n}, t \geq 0$. Projecting to the space component $\mathcal{K}$ yields $(L T 1)$ resp. $(L T 2)$

We have proved, that $(\mu(t))_{t \geq 0},\left(\lambda_{t}\right)_{t \geq 0} \subseteq \mathcal{M}_{+}(\mathcal{K})$ and have norm $\|\mu(t)\|,\left\|\lambda_{t}\right\| \leq 1$. Comparing the norms in (LT1) and (LT2) yields again that $\mu(t)$ and hence $\lambda_{t}$ are probabilities. 
The proof of Theorem 3.2 is complete.

Remark 4.26. In the particular situation with given continuous convolution semigroup $\left(\mu_{t}\right)_{t \geq 0} \subseteq \mathcal{M}^{1}(\mathcal{K})$ it is possible to find an alternative proof for the existence of a corresponding $M$-semigroup $(\mu(t))_{t \geq 0} \subseteq$ $\mathcal{M}^{1}(\mathcal{K})$ satisfying (LT1):

The alternative proof avoids space-time semigroups and relies heavily on the fact that $\mathcal{K}$ is Abelian (this was used also before, to find an example $\mathcal{A}$ of a suitable function space with prescribed properties) and on the validity of Lévy's continuity theorem.

【Let $\widehat{\mu}_{t}=e^{t L}$ with strongly negative definite $-L: \widehat{\mathcal{K}}(\equiv \mathcal{K}) \rightarrow \mathbb{R}$. (For definitions and properties of positive and negative definite functions on hypergroups see e.g. $[5,41,20]) . L$ is a continuous function and $\mathbb{R} \ni s \mapsto T_{s} \in \operatorname{Aut}(\mathcal{K})$ is continuous. Define

$$
M(t):=\int_{0}^{t} L \circ T_{s}^{*} d s=\lim _{n \rightarrow \infty} \frac{t}{n} \sum_{k=0}^{n-1} L \circ T_{k t / n}^{*}=: \lim _{n \rightarrow \infty} M_{n}(t)
$$

(where $T_{s}^{*}$ denotes the dual automorphism acting on $\widehat{\mathcal{K}}(\cong \mathcal{K})$ ).

Obviously, $M_{n}(\cdot)$ are continuous and $-M_{n}(\cdot)$ are strongly negative definite functions with corresponding continuous convolution semigroups $\left(\rho_{t}^{(n)}:=\underset{k=0}{\star} \mu_{k, t}^{n-1}\right)_{t \geq 0}$, where $\mu_{k, t}^{(n)}:=T_{k t / n}\left(\mu_{t / n}\right), \widehat{\rho_{t}^{(n)}}=e^{M_{n}(t)}$. Moreover, $e^{M_{n}(t)} \stackrel{n \rightarrow \infty}{\longrightarrow} e^{M(t)}$ (for all $t \geq 0$ ), and the limit is continuous at $e$. Therefore, according to Lévy's continuity theorem for hypergroups, there exist probabilities $\mu(t) \in \mathcal{M}^{1}(\mathcal{K})$ with $\widehat{\mu(t)}=e^{M(t)}$ and, since by construction, $t \mapsto M(t)$ is continuous, $t \mapsto \underset{n-1}{\mu(t)}$ is weakly continuous. Furthermore, by construction, $\mu(t)=\lim _{n \rightarrow \infty} \star T_{k t / n}\left(\mu_{t / n}\right)$. I.e., ( $\left.L T 1\right)$ holds. And in addition, $\forall s, t \geq 0, M(s+t)=M(s)+M(t) \circ T_{s}^{*}$. Hence $(\mu(t))_{t \geq 0}$ is a M-semigroup.

Note that by construction, $t \mapsto M(t)=\int_{0}^{t} L \circ T_{s}^{*} d s$ is differentiable in $t=0$ with $\left.\frac{d^{+}}{d t}\right|_{t=0} M(t)=L$.

On the other hand, if $\left(^{*}\right)$ is assumed for strongly negative definite functions $-M(t), t \geq 0$, and $-L: \widehat{\mathcal{K}} \rightarrow \mathbb{R}$ is continuous and strongly negative definite, then there exist $\mu(t) \in \mathcal{M}^{1}(\mathcal{K})$ and a continuous convolution semigroup $\left(\mu_{t}\right)_{t \geq 0}$, such that $(L T 2)$ holds.

【In fact, $\widehat{\mu(t / n)}^{n}=e^{t \cdot \frac{n}{t} \cdot M\left(\frac{t}{n}\right)} \rightarrow e^{t \cdot L}$. Lévy's continuity theorem proves the assertion $(L T 2): \quad \mu(t / n)^{n} \rightarrow \mu_{t}$. \

As Fourier transforms on $\widehat{\mathcal{K}}$ are real valued, it is easily shown that $(L T 2)$ is equivalent to the differentiability condition $\left(^{*}\right)$.

Hence we obtain:

Remark 4.27. The proof of (LT1) and (LT2) in Proposition 4.25 shows in view of Theorem 3.2 that for any $M$-semigroup on $\mathcal{K}$ with Fourier transform $\widehat{\mu(t)}=e^{M(t)}, t \geq 0$, the logarithms $M(t)$ are differentiable at $t=0$ and $\left.\frac{d^{+}}{d t}\right|_{t=0} M(t)=L$, the logarithm of the Fourier transform of the background driving Lévy process. 


\section{REFERENCES}

[1] Becker-Kern, P: Stable and semistable hemigroups: domains of attraction and self-decomposability. J. Theor. Probab. 16, 573-598 (2001)

[2] Becker-Kern, P: Random integral representation of operator-semi-selfsimilar processes with independent increments. Stoch. Processes Appl. 109, 327-344 (2004)

[3] Becker-Kern, P., Hazod, W.: Mehler hemigroups and embedding of discrete skew convolution semigroups on simply connected nilpotent Lie groups. Infinite dimensional harmonic analysis IV. Proceedings Tokyo (2007), 32- 46, World Sci. P. (2009)

[4] Bingham, N.H.: Lévy processes and selfdecomposability in finance. Prob. Math. Stat. 26, 367-378 (2006)

[5] Bloom, W., Heyer, H.: Harmonic Analysis of Probability Measures on Hypergroups. Walter de Gruyter, Berlin-New York (1995)

[6] Chernoff, P.: Product Formulas, Nonlinear Semigroups and Addition of Unbounded Operators. Mem. AMS 140 (1974)

[7] Drisch, Th., Hazod, W.: Analytische Vektoren von Faltungshalbgruppen I Math. Z. 172, 1-28 (1980).

[8] Duflo, M.: Représentations de semi-groupes de mesures sur un groupe localement compact. Ann. Inst. Fourier 28/3, 225-249 (1978)

[9] Duflo, M.: Semigroups of complex measures on a locally compact group. NonCommutative Harmonic Analysis: Lecture Notes Math. 466, 56-64 Springer (1975)

[10] Faraut, J.: Semigroupe de mesures complexes et calcul symbolique sur les générateurs infinitésimaux de semigroupes d'opérateurs. Ann. Inst. Fourier 20, 235-301 (1970)

[11] Faraut, J.: Semigroupe de mesures complexes sur un espace homogène et distributions dissipatives. Symposia Math. XXI, 257-265, A.P. (1977)

[12] Faraut, J., Harzallah, K.: Semigroupes d'opérateurs invariants et opérateurs dissipatifs invariants. Ann Math. Inst. Fourier 22, 147-164 (1972)

[13] Hazod, W.: Stetige Faltungshalbgruppen von Wahrscheinlichkeitsmaßen und erzeugende Distributionen. Lecture Notes in Mathematics 595. Springer (1977)

[14] Hazod, W., Siebert, E.: Stable Probability Measures on Euclidean Spaces and on Locally Compact Groups. Structural Properties and Limit Theorems. Mathematics and its Applications vol. 531. Kluwer A.P. (2001)

[15] Hazod, W.: On some convolution semi- and hemigroups appearing as limit distributions of normalized products of group-valued random variables. In: Analysis on infinite-dimensional Lie groups, Marseille (1997), H. Heyer, J. Marion ed. 104 - 121. World Sci. P. (1998)

[16] Hazod, W.: On Mehler semigroups, stable hemigroups and selfdecomposability. In: Infinite dimensional harmonic analysis III. Proceedings 2003. H. Heyer, T. Hirai, T. Kawazoe, K. Saito ed. , pp 83-98, Word Sci. P. (2005)

[17] Hazod, W.: Probability on matrix-cone hypergroups: Limit theorems and structural properties (2008) (Submitted.) (Preprint Nr. 2008-13 in: tudortmund.de/MathPreprints )

[18] Heyer, H.: Probability Measures on Locally Compact Groups. BerlinHeidelberg-New York Springer (1977)

[19] Heyer, H.: Semi-groupes de convolution sur un groupe localement compact et applications a la théorie des probabilités. Ecole d'eté de probabilités de SaintFlour VII. Lecture Notes Math. 678, 173-136, Springer (1978)

[20] Heyer, H.: Positive and negative functions on a hypergroup and its dual Infinite dimensional harmonic analysis IV. Proceedings Tokyo (2007), 63- 96, World Sci. P. (2009)

[21] Hirsch, F.: Opérateurs dissipatifs et codissipatifs invariants par translation sur les groupes localement compacts. Séminaire de théorie de potentiel. $15^{i e ̀ m e}$ année $(1971 / 72)$

[22] Hirsch, F.: Sur les semi-groupes d'opérateurs invariants par translation. CRAS Paris 274, 43-46 (1972)

[23] Hirsch, F., Roth, J.P.: Opérateurs dissipatifs et codissipatifs invariants par translation sur un espace homogène. In: Lecture Notes in Math. 404, 229-245, Springer (1974) 
[24] Hirsch, F., Roth, J.P.: Opérateurs dissipatifs et codissipatifs invariants sur un espace homogène. CRAS Paris 274, 1791-1793 (1972)

[25] Jeanblanc, M., Pitman, J., Yor, M.: Selfsimilar processes with independent increments associated with Lévy Bessel processes. Stochastic Process. Appl. 100, 223-231 (2002)

[26] Jurek, Z., Mason, D.: Operator Limit Distributions in Probability Theory, J. Wiley Inc. (1993).

[27] Jurek, Z.: An integral representation of operator- selfdecomposable random variables. Bull. Acad. Polon. Sci. 30, 385-393 (1982)

[28] Jurek, Z., Vervaat, W.: An integral representation for self-decomposable Banach space valued random variables. Z. Wahrscheinlichkeitstheorie verw. Geb. 62, $247-262$ (1983)

[29] Jurek, Z.: Self-decomposability: an exception or a rule? Annales Univ. M. Curie-Sklodowska, Lublin. Sectio A, special volume 174-182 (1997)

[30] Kunita H.: Stochastic processes with independent increments in a Lie group and their self similar properties. In: Stochastic differential and difference equations. Proceedings Györ (1996). Progress Syst. Control Theory 23, 183-201 (1997)

[31] Lumer, G., Philipps, R.S.: Dissipative operators in a Banach space. Pac. J. Math. 11, 679-698 (1961)

[32] Maejima, M., Sato, K.I..: Semi Lévy processes, self similar additive processes and semi stationary Ornstein-Uhlenbeck type processes. J. Math. Kyoto Univ. 43, 609-639 (2003)

[33] Menges, S.: Stetige Faltungshalbgruppen und Grenzwertsätze auf Hypergruppen. Dissertation, Universität Dortmund (2003)

[34] Roth, J.P.: Opérateurs dissipatifs et semi-groupes dans les espaces des fonctions continues. Ann. Inst. Fourier 26/4, 1-97 (1973)

[35] Roth, J.P.: Sur les semi-groupes à contraction invariants sur un espace homogène. C.R.Acad. Sc. Paris 227, Sér. A, 1091-1094 (1973)

[36] Rösler, M.: Convolution algebras on matrix cones. Compos. Math. 143, 749779 (2007)

[37] Sato, K.: Stochastic integrals in additive processes and application to semiLévy processes. Osaka J. Math. 41, 211-236 (2004)

[38] Sato, K., Yamazato, M.: Operator- selfdecomposable distributions as limit distributions of processes of Ornstein-Uhlenbeck type. Nagoya Math. J. 97, 71-94 (1984)

[39] Urbanik, K: Lévy's probability measures on Euclidean spaces. Studia Math. 44, 119-148 (1972)

[40] Voit, M.: Bessel convolutions on matrix cones: Algebraic properties and random walks. (2008). To appear in: J. Theor. Probab. (Preprint Nr. 2008-13 in: tu-dortmund.de/MathPreprints )

[41] Voit, M.: Positive and negative definite functions on the dual space of a commutative hypergroup. Analysis 9, 371-387 (1989)

[42] Zeuner, H.M.: Complex Lévy measures. In: Probability Measures on Groups VII. Proceedings Oberwolfach (1983), H. Heyer ed. Springer Lecture Notes Math. 1064, 471-480 (1984)

[43] Zeuner, H.M.: The Lévy Khintchine-formula for dissipative distributions, Math. Ann. 274, 273-282 (1986).

Wilfried Hazod, Faculty of Mathematics, Technische Universität Dortmund, D-44221 Dortmund, Germany

E-mail address: wilfried.hazod@math.uni-dortmund.de 


\section{Preprints ab 2008}

2008-01 Henryk Zähle

Weak approximation of SDEs by discrete-time processes

2008-02 Benjamin Fine, Gerhard Rosenberger

An Epic Drama: The Development of the Prime Number Theorem

2008-03 Benjamin Fine, Miriam Hahn, Alexander Hulpke, Volkmar große Rebel, Gerhard Rosenberger, Martin Scheer

All Finite Generalized Tetrahedron Groups

2008-04

2008-05

2008-06

Ben Schweizer

Homogenization of the Prager model in one-dimensional plasticity

Benjamin Fine, Alexei Myasnikov, Gerhard Rosenberger

Generic Subgroups of Group Amalgams

Flavius Guiaş

Generalized Becker-Döring Equations Modeling the Time Evolution of a Process of Preferential Attachment with Fitness

2008-07 Karl Friedrich Siburg, Pavel A. Stoimenov

A scalar product for copulas

2008-08 Karl Friedrich Siburg, Pavel A. Stoimenov

A measure of mutual complete dependence

2008-09 Karl Friedrich Siburg, Pavel A. Stoimenov

Gluing copulas

2008-10 Peter Becker-Kern, Wilfried Hazod

Mehler hemigroups and embedding of discrete skew convolution semigroups on simply connected nilpotent Lie groups

2008-11 Karl Friedrich Siburg

Geometric proofs of the two-dimensional Borsuk-Ulam theorem

2008-12 Michael Lenzinger and Ben Schweizer

Two-phase flow equations with outflow boundary conditions in the hydrophobic-hydrophilic case

2008-13 Wilfried Hazod

Probability on Matrix-Cone Hypergroups: Limit Theorems and

Structural Properties

2008-14 Wilfried Hazod

Mixing of generating functionals and applications to (semi-)stability

of probabilities on groups 
Wilfried Hazod

Multiple selfdecomposable laws on vector spaces and on groups:

The existence of background driving processes

2008-16 Guy Bouchitté and Ben Schweizer

Homogenization of Maxwell's equations with split rings

2008-17 Ansgar Steland and Henryk Zähle

Sampling inspection by variables: nonparametric setting

2008-18 Michael Voit

Limit theorems for radial random walks on homogeneous spaces with growing dimensions

2008-19

Michael Voit

Central Limit Theorems for Radial Random Walks on

$p \times q$ Matrices for $p \rightarrow \infty$

2008-20 Margit Rösler and Michael Voit

Limit theorems for radial random walks on $p \times q$-matrices as

$p$ tends to infinity

2008-21 Michael Voit

Bessel convolutions on matrix cones: Algebraic properties and

random walks

2008-22 Michael Lenzinger and Ben Schweizer

Effective reaction rates of a thin catalyst layer

2008-23 Ina Kirsten Voigt

Voronoi Cells of Discrete Point Sets

2008-24 Karl Friedrich Siburg, Pavel A. Stoimenov

Symmetry of functions and exchangeability of random variables

2008-25 Winfried Hazod

MEHLER SEMIGROUPS, ORNSTEIN-UHLENBECK PROCESSES

AND BACKGROUND DRIVING LÉVY PROCESSES ON LOCALLY

COMPACT GROUPS AND ON HYPERGROUPS 